\title{
OPTIMAL DYNAMIC MECHANISM DESIGN WITH DEADLINES
}

\author{
KONRAD MIERENDORFF
}

\begin{abstract}
A seller maximizes revenue from selling an object in a dynamic environment, with buyers that differ in their patience: Each buyer has a privately known deadline for buying and a privately known valuation. First, we derive the optimal mechanism, neglecting the incentive constraint for the deadline. The deadline of the winner determines the time of the allocation and therefore also the amount of information available to the seller when he decides to whether to allocate to a buyer. Depending on the shape of the markup that the seller uses, this can lead to a violation of the neglected incentive constraint. We give sufficient conditions on the type distribution under which the neglected constraint is fulfilled or violated. Second, for the case that the constraint cannot be neglected, we consider a model with two periods and two buyers. Here, the optimal mechanism is implemented by a fixed price in period one and an asymmetric auction in period two. The asymmetry, which is introduced to prevent the patient type of the first buyer from buying in period one leads to pooling of deadlines at the top of the type space.

Keywords: Dynamic Mechanism Design, Multidimensional Signals, Revenue Maximization, Deadlines

JEL-Codes: D44, D82
\end{abstract}

\section{INTRODUCTION}

In many situations, sellers face a changing population of heterogeneous buyers. Buyers arrive at different points in time. Some are impatient and want to buy immediately, others are patient and willing to wait. Patient buyers can act strategically and use their flexibility with respect to the time of a purchase in order to get better prices. Typical examples are online auctions, the sale of flight tickets, hotel reservations, or the sale of houses.

To capture heterogeneity in the degree of patience, we assume that buyers have idiosyncratic deadlines. A deadline can be viewed as an extreme form of time preferences, as in the case of a traveler who needs to buy tickets before a certain date in order to coordinate with other travel arrangements. Deadlines may also be imposed by third parties. Consider for example a company that needs to buy an input from a seller in order to

Date: July 14, 2015 (first draft June 22, 2009).

I greatly benefited from many conversations with Benny Moldovanu and Philippe Jehiel. I wish to thank the editor Alessandro Pavan, and associate editor and two anonymous referees for suggestions that greatly improved the paper and Yeon-Koo Che, Olivier Compte, Jacob Goeree, Paul Heidhues, Alexey Kushnir, Luke Lindsay, Susanne Ohlendorf, Dezsö Szalay, Alexander Westkamp, and seminar participants at Cologne, Bonn, Mannheim, Zürich, Paris, CORE, the Hausdorff Center Workshop "Information and Dynamic Mechanism Design", ESEM 2009, Yonsei, EARIE 2011 and the Arne Ryde Lectures on Dynamic Mechanism Design for helpful comments and discussions. I started this project while visiting Paris School of Economics, which I would like to thank for its hospitality. Financial support of the Bonn Graduate School of Economics, the German Academic Exchange Service and the European Research Council (ERC Advanced Investigator Grant, ESEI-249433) is gratefully acknowledged.

Contact: Department of Economics, Chair of Organizational Design, University of Zürich, Blümlisalpstrasse 10, CH-8006 Zürich, email: konrad.mierendorff@econ.uzh.ch, phone: +41 (0)446345563. 
enter a contractual relationship with a third party. This input could be a physical object, an option contract, a license, a patent, etc. It is conceivable that the third party sets a deadline, after which the contractual relationship is no longer available. Therefore, the input is worthless for the company if it is purchased after the deadline.

This paper analyzes the implications of private information about patience (deadlines) on the revenue maximizing mechanism. We consider the allocation of a single object over a finite time horizon with randomly arriving buyers and independent private values. To focus on the effects of private information about time preferences, we assume that arrival times are observable for the seller. ${ }^{1}$ Consumption is assumed to take place at the end of the time horizon (e.g., when the plane takes off and not when the ticket is sold), so that we can ignore discounting as long as all discount rates are identical.

If we relax the incentive constraint for the deadline, revenue maximization is equivalent to maximizing expected virtual surplus, subject to monotonicity with respect to the valuation. Virtual surplus maximization is a straightforward dynamic programming problem which yields the relaxed solution. Since it is costless to delay an allocation and waiting for more buyers to arrive can improve revenue, the object is allocated only at the deadline of the winner. We show that the relaxed solution can be implemented by a payment rule that applies a markup to the critical virtual valuation of the winning buyer. ${ }^{2}$ The latter is the lowest virtual valuation with which a buyer can win, given the types of all competing buyers and the seller's expectation of future arrivals. It can be interpreted as the virtual opportunity cost of awarding to the winning buyer.

The first contribution of the paper is a regularity condition under which the relaxed solution satisfies the neglected incentive constraint for the deadline. ${ }^{3}$ We show a martingale property: Increasing the deadline leads to a mean-preserving spread of the virtual opportunity. Intuitively, a later deadline allows the seller to gather more information before allocating to a buyer, which increases the dispersion of the virtual opportunity cost. The martingale property is important, because for most distributions, the seller's markup is non-linear which induces endogenous risk-preferences in the buyers. If the markup is convex, buyers become endogenously risk-averse with respect to the seller's virtual opportunity cost. If it is concave, buyers become endogenously risk-loving. Moreover, buyers can choose the dispersion of the virtual opportunity cost by choosing the reported deadline. Therefore, the shape of the markup determines whether a buyer prefers to report an earlier deadline (low dispersion) or a later deadline (high dispersion). A concave markup induces a buyer to report the latest possible deadline. He will thus report his deadline truthfully, because over-reporting would lead to an allocation after the true deadline. Formulated in terms of the distribution function, the regularity condition requires convexity of the inverse hazard rate of the valuation. Conversely, the relaxed solution is not implementable if the inverse hazard rate is concave.

\footnotetext{
${ }^{1}$ The case of unobservable arrivals is considered in Pai and Vohra (2013).

${ }^{2}$ Payoff equivalence implies that our results apply for any implementation of the relaxed solution.

${ }^{3}$ For simplicity we consider the case that the valuation and deadline of a buyer are independent. Section 3.4 discusses the correlated case.
} 
Unfortunately, examples for both the concave and the convex case abound and we cannot conclude that for most commonly used distributions, the model is regular. The second contribution of the paper is to derive an optimal mechanism in the irregular case. We consider a special case with two buyers who arrive in two different periods. The mechanism design problem becomes multi-dimensional, but the fact that the second dimension is a deadline provides some structure. The deadline enters the buyers' preferences only as a constraint. As long as a buyer gets the object before his deadline, his utility is unaffected by the deadline. This allows to formulate the seller's problem as virtual surplus maximization subject to the constraint that the utility of a buyer is non-decreasing in the deadline.

Formally, this constraint is equivalent to a type-dependent participation constraint: $\mathrm{Pa}$ tient/strategic buyers have the "outside option" to buy before their deadlines. This paper is the first so solve an optimal auction problem with a type-dependent participation constraint. The optimal control techniques employed here and the solution resemble Jullien (2000), who studies a principal-agent problem with a type-dependent (exogenous) participation constraint, but there are several notable differences. First, an auction model involves an additional feasibility constraint for the allocation rule. This is incorporated in the control problem using a characterization of asymmetric reduced form auctions (Mierendorff, 2011). Second, in an auction model, the optimal allocation rule may be discontinuous (at the reserve price). Third, we explicitly show how "ironing" is used to handle bunching in the valuation dimension because the standard assumption of a monotone virtual valuation does not imply monotonicity in the presence of type-dependent participation constraints. Forth, in contrast to Jullien (2000), the type dependent participation constraint is determined endogenously by the mechanism because the "outside option" of the patient buyer is given by the utility of an impatient buyer. We show that in the $2 \mathrm{x} 2$ model, under a mild regularity condition, the endogenous participation constraint only binds for the highest type, and then solve the model using two separate mechanism design problems. The technical reasons for the restriction to the $2 \times 2$ model, as well as possible generalizations and applications in other settings are discussed in Section 5.

In the optimal mechanism, the seller posts a fixed price in the first period. If buyer one does not accept, the seller waits for the second period and conducts an auction. If buyer one is patient, both buyers will participate in the auction. The seller has two instruments to prevent the patient/strategic type of buyer one from choosing the fixed price. He can increase the fixed price, and he can distort the auction format in the second period in favor of buyer one. Both instruments increase the expected payoff from the auction compared to the fixed price and thereby reduce the incentive for the patient/strategic type to deviate. We show that it is optimal for the seller to use both instruments. The optimal allocation rule is thus biased against impatient buyers and rewards patient buyers with a higher chance of winning. The most striking implication is that for high valuations, the mechanism no longer separates buyers with different deadlines: Buyers with low valuations have to wait until their deadline before they make a purchase. Buyers with higher valuations, on the other hand, do not benefit from waiting and may buy earlier. 
1.1. Related Literature. The literature on dynamic revenue maximization can be broadly divided into two strands. On the one hand, there are models where all buyers are impatient and therefore non-strategic with respect to the purchase time. ${ }^{4}$ This is a standard assumption in the classic revenue management literature. ${ }^{5}$ On the other hand, there are models in which all buyers are assumed to be patient and strategic. Most of this literature adopts a generalization of the standard framework with one-dimensional private information. (See Board and Skrzypacz (2015), for a model with persistent types and Pavan et al. (2014), for a general model with arrival of new information in each period.) In these models, a buyer can influence the timing of the allocation by mimicking buyers with different valuations. Moving to the multi-dimensional model allows us to study the timing decision of a buyer independently of the incentives to reveal the valuation. We show that the incentives to reveal the deadline are influenced by the markup, which reflects the seller's desire to reduce information rents arising from the private valuation. This interaction drives our new insights about the incentives to reveal time preferences and the consequences for revenue-maximization, which are obscured in a one-dimensional model.

The model analyzed in this paper has also been studied by Pai and Vohra (2013), who allow for private information about the arrival time and do not make restrictions on the number of periods or objects. ${ }^{6}$ Pai and Vohra focus on sufficient conditions for incentive compatibility of the relaxed solution. They observe that monotonicity of the winning probability of a buyer, in the deadline and the arrival time, is sufficient for incentive compatibility of the relaxed solution. For the arrival time, they show that monotonicity is guaranteed if the conditional hazard rate of the valuation is monotone in the arrival time. For the deadline, however, no sufficient condition for monotonicity of the allocation rule is available. In Appendix C.5 in the Supplemental Material, we discuss this further and show why monotonicity generally fails unless the support of the valuation distribution shifts with the deadline. In contrast to Pai and Vohra, we do not try to derive a sufficient condition via monotonicity of the winning probability. Instead, we show that the deadline of a buyer affects the amount of information available to the seller when he has to decide whether the buyer is awarded or not. The endogenous risk preferences in the buyers, arising from the seller's desire to reduce information rents, leads to our sufficient condition in terms of the shape of the hazard rate of the valuation.

This paper is also related to a literature on static mechanism design with two-dimensional private information, in which the second dimension is for example a budget constraint, a minimal capacity, or a quality constraint. ${ }^{7}$ In these models, the second dimension of

${ }^{4}$ See for example Das Varma and Vettas (2001); Vulcano et al. (2002); Gershkov and Moldovanu (2009a); Dizdar et al. (2011).

${ }^{5}$ See Elmaghraby and Keskinocak (2003) for a survey. McAfee and te Velde (2007) survey airline pricing. $\mathrm{Su}(2007)$ studies a model with patient buyers.

${ }^{6}$ Our sufficient condition also holds with many objects and two time periods.

${ }^{7}$ See Beaudry et al. (2009) for an analysis of optimal taxation; Blackorby and Szalay (2008) and Szalay (2009) for regulation; Iyengar and Kumar (2008) and Dizdar et al. (2011) for auction models with capacitated bidders; Che and Gale (2000), Malakhov and Vohra (2005) and Pai and Vohra (2014) for models with budget constrained buyers. 
private information has a special structure similar to the deadline in our model, which makes the analysis tractable. ${ }^{8}$ With the exception of Szalay (2009), who studies a singleagent model, this literature typically makes assumptions on the correlation between the two dimensions which guarantee that the relaxed solution is incentive compatible. We add to this literature by deriving the optimal mechanism in the irregular case.

This paper differs from the literature on dynamic screening in which buyers learn about their valuations over time (Courty and Li, 2000; Eso and Szentes, 2007; Pavan et al., 2014). This literature studies the case where agents receive (one-dimensional) private information in each period but types evolve over time. ${ }^{9}$ Instead, we consider a model with persistent types where private information is two-dimensional, which allows for richer time preferences. Several papers have considered efficient dynamic mechanism design (see Parkes and Singh, 2003; Bergemann and Välimäki, 2010; Athey and Segal, 2013). The incentive problems analyzed in the present paper disappear for value-maximization because the seller does not use a markup and the relaxed solution is always incentive compatible.

Organization of the Paper. Section 2 describes the model and formulates the seller's problem. Section 3 presents the relaxed solution and conditions for incentive compatibility, formal proofs are in Appendix A. Section 4 presents the general solution for the irregular case. The formal derivation is in Appendix B. Section 5 concludes. Appendix C in the Supplemental Material in contains some omitted proofs, discussions, and an extension of Section 3 to multiple objects.

\section{The Model}

Consider a seller who wants to maximize the expected revenue from selling an indivisible object within $T<\infty$ time periods. In the case of an airline ticket, $T$ is the time when the plane takes off. The seller's valuation (or production cost) is normalized to zero. In each period, a random number of buyers $N_{t} \geq 0$ arrives. The set of buyers who arrive in period $t$ is denoted $I_{t} . I_{\leq t}:=\bigcup_{\tau=1}^{t} I_{\tau}$ and $N_{\leq t}:=\left|I_{\leq t}\right|$ describe the arrivals until period $t$. A buyer $i \in I_{t}$ is characterized by his arrival time $a_{i}=t$, his valuation $v_{i} \in[0, \bar{v}]$, where $\bar{v}>0$, and his deadline $d_{i} \in\{t, \ldots, T\}$. The object cannot be sold to a buyer before his arrival time. Utility is quasi-linear. If buyer $i$ has to make a total payment of $y_{i}$, then his total payoff is $v_{i}-y_{i}$ if he gets the object in periods $a_{i}, \ldots, d_{i}$, and $-y_{i}$ otherwise. $^{10}$

\footnotetext{
${ }^{8}$ The models of Rochet and Choné (1998), Jehiel et al. (1999), and others, in which all dimensions are symmetric, rarely have explicit solutions (see Armstrong, 1996, for an exception).

${ }^{9}$ See also Battaglini (2005), Nocke and Peitz (2007), Möller and Watanabe (2010), Deb (2011) and references therein. For surveys of the literature on dynamic mechanism design see Bergemann and Said (2011), and Gershkov and Moldovanu (2012a). Gershkov and Moldovanu (2009b, 2012b, 2010) have studied dynamic mechanism design problems in which the seller learns about future buyers' type distributions from current buyers' types. We abstract from learning by the seller and assume that types are uncorrelated.

${ }^{10}$ Note that the deadline is part of the preferences of a buyer. An alternative interpretation would be that the deadline is the time when the buyer will exit the market. This interpretation restricts the class of mechanism that the seller can use, because payments would have to take place before the deadline of a buyer. It turns out, however, that the optimal mechanism does not require payments after the deadline. Therefore, both interpretations of the deadline lead to the same results.
} 
Buyers are risk-neutral and maximize expected payoff. Neither the buyers nor the seller discount future payoffs. ${ }^{11}$

The numbers of arrivals in different periods and the types of different buyers are independently distributed. Moreover, to focus on the novel insights that arise due to the dynamic structure of the model, we assume that conditional on the arrival time, the deadline and the valuation of a buyer are independent. ${ }^{12} \nu_{t}\left(N_{t}\right)$ denotes the probability that $N_{t}$ buyers arrive in period $t$. To exclude uninteresting cases, we assume that in each period, there is a positive probability of new arrivals $\left(\forall t: \nu_{t}(0)<1\right)$. For a given arrival time $a$, the probability that the deadline of a buyer equals $d$ is denoted $\rho_{a}(d)$. The valuation has distribution function $F_{a}(v)$ and density $f_{a}(v) .{ }^{13}$

Information realizes over time. In period $t$, the numbers of future buyers $N_{t+1}$, $\ldots, N_{T}$, and their types are not known to anybody. In particular, the decision to sell a unit in period $t$ cannot be based on this information. Upon arrival, each buyer privately observes his valuation and his deadline. In order to focus on the incentive issues of private information about deadlines, we assume that the seller observes arrivals. ${ }^{14} \nu_{t}(\cdot), \rho_{a}(\cdot)$ and $F_{a}(\cdot)$ are commonly known.

Finally, we assume that for all $a, f_{a}(v)$ is continuous in $v$ and strictly positive for all $v \in[0, \bar{v}]$, continuously differentiable in $v$ for $v \in(0, \bar{v})$, and that $f_{1}^{\prime}($.$) can be extended$ continuously to $[0, \bar{v}]$. To avoid additional technicalities, the following standard regularity condition is imposed throughout the paper.

Assumption 1. For all $a \in\{1, \ldots T\}$, the virtual valuation $J_{a}(v):=v-\frac{1-F_{a}(v)}{f_{a}(v)}$ is strictly increasing in $v$.

For some results in Section 4, we will assume that the monopoly profit from selling to a single buyer in the first period is concave:

Assumption 2. $v\left(1-F_{1}(v)\right)$ is concave.

Assumption 2 is equivalent to the assumption that $J_{1}(v) f_{1}(v)$ is increasing.

2.1. Allocation and Payment Rules. In order to describe allocation and payment rules in this dynamic setting we define a state as $s_{t}=\left(H_{t}, k_{t}\right) . H_{t}=\left(a_{i}, v_{i}, d_{i}\right)_{i \in I_{\leq t}}$ denotes the history of buyer types that have arrived so far, and $k_{t}$ indicates whether the object is still available $\left(k_{t}=1\right)$, or has already been allocated $\left(k_{t}=0\right)$. The history of buyer types excluding $i$ is denoted $H_{t}^{-i}$.

\footnotetext{
${ }^{11}$ If only payments are discounted and all agents have a common discount factor, the results do not change. The assumption that the value of consumption is not discounted is natural in the settings discussed in the introduction were consumption takes place at time $T$, independent of the time of the allocation (e.g. of a ticket or a reservation). In other applications, discounting may be more natural.

${ }^{12}$ In Section 3.4, we will discuss the consequences of correlations between the deadline and the valuation.

${ }^{13}$ To simplify notation, we assume that buyers with the same arrival period are ex-ante identical. All results carry over to the case of ex-ante asymmetric bidders.

${ }^{14}$ See Pai and Vohra (2013) for a discussion of private information about arrival times.
} 
An allocation rule defines a winning probability $x_{i}\left(s_{t}\right) \in[0,1]$ for each state $s_{t}$, and for each buyer $i \in I_{\leq t}$. An allocation rule must satisfy the feasibility constraint

$$
\forall t, s_{t}: \quad \sum_{i \in I_{\leq t}} x_{i}\left(s_{t}\right) \leq k_{t} .
$$

The probability that the object is not allocated in state $s_{t}$ is denoted by $x_{0}\left(s_{t}\right)=k_{t}-$ $\sum_{i \in I_{\leq t}} x_{i}\left(s_{t}\right)$. An allocation rule allocates only at the deadline if $x_{i}\left(s_{t}\right)=0$ for all $t, s_{t}$ and all $i \in I_{\leq t}$ with $d_{i} \neq t$. A payment rule defines a payment $y_{i}\left(s_{t}\right)$ for each buyer $i \in I_{\leq t}$ in every state $s_{t} \cdot{ }^{15}$ It will be without loss for the seller's maximal profit to consider only symmetric allocation and payment rules, i.e., rules that do not discriminate between buyers with the same arrival times. ${ }^{16}$

2.2. Mechanisms. The seller's goal is to design a mechanism that has a Bayes-Nashequilibrium which maximizes expected revenue. In general, a mechanism can be any game form with $T$ stages, such that only buyers from $I_{\leq t}$ are active in stage $t$. We assume that the mechanism designer has full commitment power and can choose to conceal any information about the first $t$ stages from the buyers that arrive in stages $t+1, \ldots, T .{ }^{17}$

By the revelation principle, the seller can restrict attention to incentive compatible and individually rational direct mechanisms, in which no information is revealed. ${ }^{18}$ Furthermore, we impose symmetry as discussed above.

Definition 1. A direct mechanism consists of message spaces $S_{1}=[0, \bar{v}] \times\{1, \ldots, T\}$, $\ldots, S_{T}=[0, \bar{v}] \times\{T\}$, and symmetric allocation and payment rules $(x, y)$.

For a given direct mechanism $(x, y)$, consider a buyer $i \in I_{a}$ who reports $(v, d) \in S_{a}$. If all other buyers (past, current and future) report their types truthfully, the interim winning probability - that is, the probability that this buyer wins in period $t \geq a$, is given by

$$
q_{a}^{t}(v, d):=E\left[x_{i}\left(s_{t}\right) \mid\left(a_{i}, v_{i}, d_{i}\right)=(a, v, d)\right] .
$$

The interim expected payment is given by

$$
p_{a}(v, d):=E\left[\sum_{\tau=a}^{T} y_{i}\left(s_{\tau}, k_{\tau+1}\right) \mid\left(a_{i}, v_{i}, d_{i}\right)=(a, v, d)\right] .
$$

\footnotetext{
${ }^{15}$ Here, we implicitly assume that the payment in period $t$ only depends on $s_{t}$. Since we will consider Bayesian incentive compatibility and interim participation constraints in what follows, this is without loss of generality.

${ }^{16}$ Formally, an allocation rule (payment rule) is called symmetric if for all $t$, all states $s_{t}$, and all $i, j \in I_{\leq t}$ such that $a_{i}=a_{j}: x_{i}\left(s_{t}\right)=x_{j}\left(\sigma_{i, j}\left(H_{t}\right), k_{t}\right)\left(y_{i}\left(s_{t}\right)=y_{j}\left(\sigma_{i, j}\left(H_{t}\right), k_{t}\right)\right)$, where $\sigma_{i, j}$ denotes the permutation that interchanges the $i^{\text {th }}$ and the $j^{\text {th }}$ element of its argument.

${ }^{17}$ This assumption yields an upper bound on the revenue that can be achieved. We will see that this bound can also be achieved in a periodic ex-post equilibrium, i.e., if buyers observe all information from past and current stages.

${ }^{18}$ The standard revelation principle holds because the seller observes arrival times. Note that without this assumption, each buyer could mimic all types with an arrival time greater or equal than his own arrival time. Therefore, the nested range condition is satisfied and the revelation principle holds (see Green and Laffont, 1986; Bull and Watson, 2007).
} 
Note that we aggregate payments from different periods in this definition. ${ }^{19}(q, p)$ is called the reduced form of $(x, y)$. The interim expected utility from participating in a mechanism $(x, y)$, for a buyer with true type $(v, d)$ who reports $\left(v^{\prime}, d^{\prime}\right)$, is given by

$$
U_{a}\left(v, d, v^{\prime}, d^{\prime}\right):=\left[\sum_{\tau=a}^{d} q_{a}^{\tau}\left(v^{\prime}, d^{\prime}\right)\right] v-p_{a}\left(v^{\prime}, d^{\prime}\right) .
$$

The expected utility from truth-telling is abbreviated $U_{a}(v, d):=U_{a}(v, d, v, d)$.

Definition 2. (i) A direct mechanism $(x, y)$ is (Bayesian) incentive compatible if for all $a \in\{1, \ldots, T\}, v, v^{\prime} \in[0, \bar{v}]$, and $d, d^{\prime} \in\{a, \ldots, T\}$,

$$
U_{a}(v, d) \geq U_{a}\left(v, d, v^{\prime}, d^{\prime}\right) .
$$

(ii) A direct mechanism $(x, y)$ is individually rational if for all $1 \leq a \leq d \leq T$, and all $v \in[0, \bar{v}]$

$$
U_{a}(v, d) \geq 0
$$

2.3. Characterization of Incentive Compatibility. In this section we derive a basic characterization of incentive compatibility. ${ }^{20}$ We restrict attention to mechanisms that allocate only at the deadline. The following Lemma shows that in the absence discounting, this is without loss.

Lemma 1. Let $(x, y)$ be a direct mechanism that satisfies (IC) and (IR). Then, there exists an alternative mechanism $(\hat{x}, \hat{y})$ such that $\hat{x}$ allocates only at the deadline, $(\hat{x}, \hat{y})$ satisfies $(I C)$ and $(I R)$, and $(x, y)$ and $(\hat{x}, \hat{y})$ yield the same expected revenue.

Proof. The proof can be found in Appendix C.1 in the Supplemental Material

The idea behind the Lemma is that delaying an allocation until the reported deadline of a buyer does not change his payoff if he reports the true or an earlier deadline. At the same time, a buyer who reported a later deadline than his true deadline may be worse off, if his allocation is delayed to the reported deadline. Therefore, a modification of a given mechanism that moves all allocations to the reported deadlines of the respective buyers relaxes incentive constraints. Hence, the seller does not loose revenue by allocating only at the deadline. In light of Lemma 1 , we will only consider mechanisms that only allocate at the deadline and write $q_{a}(v, d)$ instead of $q_{a}^{d}(v, d)$ in what follows.

The following characterization of incentive compatibility builds on Myerson's characterization for one-dimensional private information. In addition, we have to ensure that buyers do not have an incentive to misreport the deadline. If a mechanism only allocates at the deadline, we only have to rule out downward deviations. The characterization requires that the expected utility from participating with a truthful report is weakly increasing in the deadline. For the lowest type $v=0$, we have to strengthen this condition and require

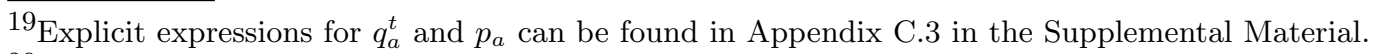

${ }^{20}$ Similar characterizations have been used in the previous literature on two-dimensional incentive problems. See for example Pai and Vohra (2013).
} 
that the utility is independent of the deadline. The following theorem shows that this one-dimensional constraint, together with Myerson's characterization is sufficient to rule out simultaneous misreports of the deadline and the valuation.

Theorem 1. Let $(x, y)$ be a direct mechanism that allocates only at the deadline, with reduced form $(q, p)$. Then $(x, y)$ is incentive compatible if and only if for all $1 \leq a \leq d \leq T$, and all $v, v^{\prime} \in[0, \bar{v}]:$

$$
\begin{aligned}
v>v^{\prime} & \Rightarrow q_{a}(v, d) \geq q_{a}\left(v^{\prime}, d\right), \\
U_{a}(v, d) & =U_{a}(0, d)+\int_{0}^{v} q_{a}(s, d) d s, \\
U_{a}(v, d) & \leq U_{a}(v, d+1), \quad \text { if } d<T, \\
\text { and } \quad U_{a}(0, d) & =U_{a}(0, d+1), \quad \text { if } d<T .
\end{aligned}
$$

Proof. The proof can be found in Appendix C.1 in the Supplemental Material

Sufficiency is implied by the special structure of preferences. The deadline is a constraint that does not affect a buyer's payoff as long as the object is awarded before the deadline. Therefore, a buyer who reports $d^{\prime}<d_{i}$ enjoys the same expected payoff as a buyer whose true deadline is $d^{\prime}$ and who reports his deadline truthfully. For such a buyer, however, $(\mathrm{M})$ and $(\mathrm{PE})$ ensure that a truthful report of the valuation is optimal. Therefore, buyer $i$ does not have an incentive to misreport both dimensions simultaneously.

Formally, the downward incentive constraint for the deadline resembles an endogenous, type-dependent participation constraint. A patient/strategic buyer with arrival time $a$ and deadline $d>a$ has the "outside option" to report $d^{\prime}<d$. He only "participates" voluntarily with a truthful report, if his payoff with $d^{\prime}=d$ exceeds the payoff of his best "outside option."

2.4. The Seller's Problem. By the revelation principle and Lemma 1, the seller's problem is to choose an incentive compatible and individually rational direct mechanism that allocates only at the deadline, to maximize

$$
\sum_{a=1}^{T}\left\{E\left[N_{a}\right] E\left[p_{a}(v, d)\right]\right\} .
$$

Using (2.1) and (PE) to substitute the payment rule, integrating by parts and setting $U_{a}(0, d)=0$ for all $1 \leq a \leq d \leq T$, the objective of the seller can be rearranged to

$$
\sum_{a=1}^{T}\left\{E\left[N_{a}\right] \sum_{d=a}^{T} \rho_{a}(d) E\left[q_{a}(v, d) J_{a}(v)\right]\right\} .
$$

Next, we substitute $q_{a}(v, d)$, and bring the seller's maximization problem into a recursive form. The resulting dynamic program is denoted $\mathcal{R}$ :

$$
V_{T}\left(s_{T}\right):=\max _{x\left(s_{T}\right)} \sum_{i \in I_{\leq T}: d_{i}=T} x_{i}\left(s_{T}\right) J_{a_{i}}\left(v_{i}\right),
$$




$$
\forall t<T: V_{t}\left(s_{t}\right):=\max _{x\left(s_{t}\right)} \sum_{i \in I_{\leq t}: d_{i}=t} x_{i}\left(s_{t}\right) J_{a_{i}}\left(v_{i}\right)+x_{0}\left(s_{t}\right) E_{s_{t+1}}\left[V_{t+1}\left(s_{t+1}\right) \mid s_{t}, k_{t+1}=1\right],
$$

where the reduced form of the optimal policy $x$ must satisfy $(\mathrm{M}),\left(\mathrm{ICD}^{\mathrm{d}}\right)$, and $(\mathrm{PE})$ where we set $U_{a}(0, d) \equiv 0$. As is common in one-dimensional auction problems, the seller chooses a policy that maximizes the expected virtual valuation of the winning buyer.

\section{The Relaxed Solution}

Once the seller's objective function has been transformed into virtual value form as in $(\mathcal{R})$, the standard approach to maximize revenue is to relax all remaining constraints except for participation and feasibility constraints. The solution to this problem is the relaxed solution $x^{\mathrm{rlx}}$. Assumption 1 ensures that $x^{\mathrm{rlx}}$ satisfies the monotonicity constraint (M). Therefore $x^{\mathrm{rlx}}$ is an optimal allocation rule if the deadlines of all buyers are public. In this section we first define a payment rule $y^{\mathrm{rlx}}$, that implements the allocation rule $x^{\mathrm{rlx}}$ under the assumption that deadlines are public, but valuations are private. The definition uses critical virtual valuations. Next, we derive the martingale property, which leads to Lemma 2. Finally we will show how this property can be used to derive sufficient conditions for incentive compatibility of the relaxed solution with respect to the deadline, which leads to our first main result in Theorem 3. Throughout this section will use the properties of one particular payment rule $y^{\text {rlx }}$. Note, however, that payoff equivalence implies that the results of this exercise, in particular Theorem 3, also apply to all other payment rules that implement $x^{\mathrm{rlx}}$, as long as $U_{a}(0, d)=U_{a}(0, d+1)$ is satisfied for all $a$ and $d$.

\subsection{A Payment Rule that Implements the Relaxed Solution with Public Dead-}

lines. We define a payment rule in which the transfer of a bidder is zero if he does not win the object. The winning bidder has to make a payment that is equal to the lowest valuation with which he can win the object for given arrivals and types of competing bidders. To define this payment rule formally, fix a buyer $i$ with arrival time $a$ and suppose that the object is still available in the arrival period $\left(k_{a}=1\right)$. We define the critical virtual valuation for all $d \geq a$ and all continuations $H_{d}^{-i}$ of $H_{a}^{-i}$ as $^{21}$

$$
\underline{J}_{a}\left(H_{d}^{-i}\right):=J_{a}\left(\inf \left\{v \geq 0 \mid x_{i}^{\mathrm{rlx}}\left(\left(H_{d}^{-i},\left(a_{i}, v_{i}, d_{i}\right)=(a, v, d)\right), k_{d}\right)=1\right\}\right) .
$$

$\underline{J}_{a}\left(H_{d}^{-i}\right)$ is called the critical virtual valuation because it is the lowest virtual valuation with which buyer $i$ with deadline $d$ wins against the other buyers that arrive until period $d$, for a given history $H_{d}^{-i}$. With this notation we can rewrite $x^{\text {rlx }}$ as follows: ${ }^{22}$

$$
x_{i}^{\mathrm{rlx}}\left(s_{t}\right):= \begin{cases}1, & \text { if } k_{a_{i}}=1, t=d_{i}, \text { and } J_{a}\left(v_{i}\right) \geq \underline{J}_{a_{i}}\left(H_{d_{i}}^{-i}\right), \\ 0, & \text { otherwise. }\end{cases}
$$

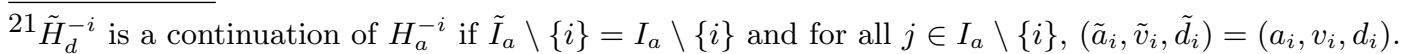

${ }^{22}$ For simplicity, we ignore ties. The subsequent analysis is valid for any deterministic tie-breaking rule. With random tie-breaking we would have to condition payments on the realized allocation decision in addition to the state. This would only complicate the notation without changing the results. 
While rewriting $x^{\mathrm{rlx}}$ in this way is tautological, (3.1) serves well to define a simple payment rule $y^{\text {rlx }}$ :

$$
y_{i}^{\mathrm{rlx}}\left(s_{t}\right):= \begin{cases}J_{a}^{-1}\left(\underline{J}_{a_{i}}\left(H_{d_{i}}^{-i}\right)\right), & \text { if } x_{i}^{\mathrm{rlx}}\left(s_{t}\right)=1, \\ 0 & \text { otherwise. }\end{cases}
$$

Theorem 2. If deadlines are public information, then $\left(x^{r l x}, y^{r l x}\right)$ is incentive compatible and maximizes the seller's revenue. Moreover, truthfully reporting the valuation is a weakly dominant strategy.

Proof. With the payment rule $y^{\text {rlx }}$, the payment of a losing buyer is zero. The winner pays the lowest valuation with which he could have obtained the object for a given history of arrivals until period $d_{i}$. Thus, truth-telling is a weakly dominant strategy if the deadline is public and buyers only report their valuations. $\left(x^{\mathrm{rlx}}, y^{\mathrm{rlx}}\right)$ is optimal because any mechanism that implements $x^{\text {rlx }}$ and satisfies $U_{a}(0, d)=U_{a}(0, d+1)$ for all $a$ and $d$, yields the same expected revenue, which follows from payoff equivalence.

3.2. The Martingale Property. The next step is to identify conditions under which the mechanism $\left(x^{\mathrm{rlx}}, y^{\mathrm{rlx}}\right)$ satisfies the incentive constraint for the deadline $\left(\mathrm{ICD}^{\mathrm{d}}\right)$. The critical virtual valuation can be interpreted as the virtual opportunity cost of an allocation to a buyer. This can be easily seen in the following example. The example also illustrates a property that is crucial for the subsequent analysis: the sequence of critical virtual valuations for different deadlines $\left(\underline{J}_{a_{i}}\left(H_{d}^{-i}\right)\right)_{d=a_{i}, \ldots, T}$ is a martingale.

Example. Let $T=2$, and $\nu_{1}(1)=\nu_{2}(1)=1$, that is, exactly one buyer arrives in each period. If the first buyer has deadline $d_{1}=1$, then he is awarded the object if his virtual valuation is greater than the option value of waiting, which equals $E_{v_{2}}\left[\max \left\{0, J_{2}\left(v_{2}\right)\right\}\right]$. Hence, the critical virtual valuation of buyer one for $d_{1}=1$ is

$$
\underline{J}_{1}\left(H_{1}^{-1}\right)=E_{v_{2}}\left[\max \left\{0, J_{2}\left(v_{2}\right)\right\}\right] .
$$

If $d_{1}=2$, the object will not be allocated in period one because there is no buyer who has reached his deadline. In period two, the virtual opportunity cost of allocating to buyer one is $\max \left\{0, J_{2}\left(v_{2}\right)\right\}$. Therefore, the critical virtual valuation of buyer one for $d_{2}=1$ is

$$
\underline{J}_{1}\left(H_{2}^{-1}\right)=\max \left\{0, J_{2}\left(v_{2}\right)\right\} .
$$

Clearly, $\left(\underline{J}_{1}\left(H_{1}^{-1}\right), \underline{J}_{1}\left(H_{2}^{-1}\right)\right)$ is a martingale because $E\left[\underline{J}_{1}\left(H_{2}^{-1}\right) \mid H_{1}^{-1}\right]=\underline{J}_{1}\left(H_{1}^{-1}\right)$.

To derive the martingale property more generally, the basic definition of the critical virtual valuation by (3.1) is not very useful. A simpler expression can be obtained by defining a score $\pi_{i}$ for each buyer, which depends only on the buyer's own type. Mierendorff (2013) shows that a score can be defined such that buyer types with different arrival times and deadlines become directly comparable. ${ }^{23}$ In particular, in each period, the allocation

\footnotetext{
${ }^{23}$ That paper considers value-maximization in a more general allocation problem. Replacing values by virtual values, however, does not change the result we use here.
} 
rule in the relaxed solution will pick the buyer with the highest (positive) score among all buyers that have arrived so far. If the deadline of this buyer is the current period, the object will be allocated to him. If his deadline is in the future, or if the highest score is negative, the object is not allocated in the current period. Formally, we will have:

$$
x_{i}^{\mathrm{rlx}}\left(s_{t}\right)= \begin{cases}1, & \text { if } k_{a_{i}}=1, t=d_{i}, \text { and } \pi_{i} \geq \max \left\{0, \max _{j \in I_{\leq d_{i}}} \pi_{j}\right\}, \\ 0, & \text { otherwise. }\end{cases}
$$

This structure also implies that if the object is still available in period $t$, then the deadline of the buyer with the highest positive score cannot be in the past. If it was in the past, then he would have gotten the object at his deadline because he had the highest score in that period already.

To define the score, we introduce an artificial state $\hat{s}_{d}(\pi)$ for a given period $d$. In this state, the object is still available $\left(\hat{k}_{d}=1\right)$, and there is a single artificial buyer $\left(\hat{I}_{\leq d}=\{1\}\right)$. The buyer has arrival time $\hat{a}_{1}=1$, virtual valuation $J_{1}\left(\hat{v}_{1}\right)=\pi$, and his deadline is $\hat{d}_{1}=T .{ }^{24}$ If $V_{d_{i}}\left(\hat{s}_{d_{i}}(0)\right) \leq J_{a}\left(v_{i}\right)$, the score of buyer $i$ is defined implicitly by

$$
J_{a}\left(v_{i}\right)=V_{d_{i}}\left(\hat{s}_{d_{i}}\left(\pi_{i}\right)\right) .
$$

If $V_{d_{i}}\left(\hat{s}_{d_{i}}(0)\right)>J_{a}\left(v_{i}\right)$, we set $\pi_{i}=-1$. Mierendorff (2013) shows that the relaxed solution coincides with the solution defined by (3.3) and (3.4).

The expected revenue in the artificial state $\hat{s}_{d_{i}}(0)$ is the same as the expected revenue in a state where no buyer is currently available, because the artificial buyer in $\hat{s}_{d_{i}}(0)$ has virtual valuation zero. If $V_{d_{i}}\left(\hat{s}_{d_{i}}(0)\right)>J_{a}\left(v_{i}\right)$ the seller would therefore never allocate to buyer $i$-in terms of virtual surplus, he is better off without buyer $i$. We assign an arbitrarily chosen negative score $(-1)$ to a buyer with such a low valuation. In the opposite case the score is non-negative. The score $\pi_{i}$ is then defined such that $i$ 's virtual value is equal to the continuation value of the seller's problem in the artificial state with a single buyer who has a virtual valuation equal to the score $\pi_{i}$ and deadline $T$. In other words, (3.4) implies that in period $d_{i}$, the seller is indifferent, in terms of virtual surplus, between allocating to buyer $i$, or switching to the artificial state $\hat{s}_{d_{i}}\left(\pi_{i}\right)$. We have thus transformed buyer $i$ 's valuation into a score that describes the equivalent (in terms of seller-revenue) virtual valuation of a buyer with deadline $T$.

(3.3) has further implications. Since the buyer with the highest score cannot loose against any buyer who has already arrived, $V_{t}\left(H_{t}, 1\right)$ only depends on the type of the buyer with the highest score. Moreover, Mierendorff (2013) shows that in any state where the object is still available, the seller's continuation value is the same as the continuation value in the artificial state $\hat{s}_{t}\left(\max \left\{0, \max _{j \in I_{\leq t}} \pi_{j}\right\}\right)$. Formally, for all $t$ and $H_{t}$ such that $x_{0}^{\mathrm{rlx}}\left(H_{t^{\prime}}, 1\right)=1$ for all $t^{\prime}<t$,

\footnotetext{
$\overline{{ }^{24} \text { We can choose }} \hat{a}_{1} \leq d$ arbitrarily. If $\hat{a}_{1} \neq 1$, then $J_{\hat{a}_{1}}\left(\hat{v}_{1}\right)=\pi$. It is important, however, that the deadline is $T$.
} 


$$
V_{t}\left(H_{t}, 1\right)=V_{t}\left(\hat{s}_{t}\left(\max \left\{0, \max _{j \in I_{\leq t}} \pi_{j}\right\}\right)\right) .
$$

Equipped with these properties of the relaxed solution, we can now study the relationship between critical virtual valuations for different deadlines. Consider a buyer $i$ who arrives in a period where the object is still available. If $i$ 's deadline is $d$, he wins in period $d$ if

$$
\pi_{i} \geq \max \left\{0, \max _{j \in I_{\leq d}} \pi_{j}\right\}
$$

Using (3.4), this yields

$$
J_{a_{i}}\left(v_{i}\right)=V_{d}\left(\hat{s}_{d}\left(\pi_{i}\right)\right) \geq V_{d}\left(\hat{s}_{d}\left(\max \left\{0, \max _{j \in I_{\leq d} \backslash\{i\}} \pi_{j}\right\}\right)\right)
$$

because the continuation value in the artificial state is weakly increasing in the artificial buyer's virtual valuation. Therefore, we have

$$
\underline{J}_{a_{i}}\left(H_{d}^{-i}\right)=V_{d}\left(\hat{s}_{d}\left(\max \left\{0, \max _{j \in I_{\leq d} \backslash\{i\}} \pi_{j}\right\}\right)\right) .
$$

We can (3.6) for $d-1$ to get

$$
\begin{aligned}
\underline{J}_{a_{i}}\left(H_{d-1}^{-i}\right) & =V_{d-1}\left(\hat{s}_{d-1}\left(\max \left\{0, \max _{j \in I_{\leq d-1} \backslash\{i\}} \pi_{j}\right\}\right)\right) \\
& =E\left[V_{d}\left(s_{d}\right)\left|s_{d-1}=\hat{s}_{d-1}\left(\max \left\{0, \max _{j \in I_{\leq d-1} \backslash\{i\}} \pi_{j}\right\}\right)\right| H_{d-1}^{-i}\right] \\
& =E\left[V_{d}\left(\hat{s}_{d}\left(\max \left\{0, \max _{j \in I_{\leq d} \backslash\{i\}} \pi_{j}\right\}\right)\right) \mid H_{d-1}^{-i}\right] \\
& =E\left[\underline{J}_{a_{i}}\left(H_{d}^{-i}\right) \mid H_{d-1}^{-i}\right] .
\end{aligned}
$$

To obtain the second line, we have used to definition of $V_{d-1}$ and the fact that $x_{0}^{\mathrm{rlx}}=1$ in the artificial state $\hat{s}_{d-1}\left(\max \left\{0, \max _{j \in I_{\leq d-1} \backslash\{i\}} \pi_{j}\right\}\right)$, which holds because the deadline of the artificial buyer is $T$. Note that in the second line, we take the expectation of $V_{d}\left(s_{d}\right)$ where $s_{d}$ is the state in which the set of buyers is given by the artificial buyer from $\hat{s}_{d-1}$, joined with the new arrivals from period $d$. To obtain the third line we have used (3.5) which applies here because the deadlines of all buyers in state $s_{d}$ are greater or equal than $d$. To obtain the last line, we have used (3.6) for $d$. To summarize, we have shown that the sequence of random variables $\left(J_{a_{i}}\left(H_{d}^{-i}\right)\right)_{d=a, \ldots, T}$ is a martingale. The following lemma slightly strengthens this result and shows strict second-order stochastic dominance of critical virtual valuations for different deadlines. ${ }^{25}$

\footnotetext{
${ }^{25} \mathrm{It}$ is well known in the literature on dynamic programming, that the stopped sequence in an optimal stopping problem is a martingale. The martingale property of the critical virtual valuation, however, does not follow from this result. For a more detailed discussion of the two properties, see Appendix C.4 in the Supplemental Material.
} 
Lemma 2. For all states $s_{a}$ with $k_{a}=1$, and all $i \in I_{a},\left(\underline{J}_{a}\left(H_{d}^{-i}\right)\right)_{d=a, \ldots, T}$ is a martingale (with respect to $\left.\left(H_{d}^{-i}\right)_{d=a, \ldots, T}\right)$ : for all $d \in\{a+1, \ldots, T\}$,

$$
E_{H_{d}^{-i}}\left[\underline{J}_{a}\left(H_{d}^{-i}\right) \mid H_{d-1}^{-i}\right]=\underline{J}_{a}\left(H_{d-1}^{-i}\right) .
$$

Furthermore, if $\underline{J}_{a}\left(H_{a}^{-i}\right)<\bar{v}$, then for all $d, d^{\prime} \in\{a, \ldots, T\}, d<d^{\prime}$

$$
\left[\underline{J}_{a}\left(H_{d}^{-i}\right) \mid s_{a}\right] \succ_{S S D}\left[\underline{J}_{a}\left(H_{d^{\prime}}^{-i}\right) \mid s_{a}\right],
$$

where $\succ_{S S D}$ denotes strict second-order stochastic dominance.

Proof. The proof of strict second-order stochastic dominance can be found in Appendix A.

The martingale property implies that while the critical virtual valuation becomes more variable if the deadline increases, its expectation is independent of the deadline, exactly as in the example. Intuitively, competition by other buyers does not become more or less intense if the deadline changes. The deadline only determines which cohorts of buyers are incorporated in the option value and for which cohorts a buyer competes against the realized virtual valuations.

3.3. Incentive Compatibility of the Relaxed Solution. The final step in this section is to use Lemma 2 to derive sufficient conditions under which $x^{\text {rlx }}$ satisfies or violates $(\mathrm{ICD})$, respectively. The main idea can be illustrated by considering $\left(\mathrm{ICD}^{\mathrm{d}}\right)$ for a buyer with the highest possible valuation $v_{i}=\bar{v}$. For any deadline $d$, we have

$$
U_{a}(\bar{v}, d)=\left(\bar{v}-E\left[y_{i}^{\mathrm{rlx}}\left(s_{d}\right) \mid k_{a}=1\right]\right) \times \operatorname{Prob}\left[k_{a}=1\right] .
$$

A buyer $v_{i}=\bar{v}$ wins with probability one whenever the object is still available at his arrival time. Therefore, the expected utilities for different deadlines $d$ and $d^{\prime}>d$ can differ only in the expected payments. The payment is defined in (3.2) by applying a markup given by $J_{a}^{-1}$ to the virtual opportunity cost of the seller. If the markup is linear, the buyer is be risk-neutral with respect to the virtual opportunity cost of the seller and hence indifferent between reporting different deadlines. If the markup is non-linear, however, the buyer becomes endogenously risk-averse or risk-loving. For example, if $J_{a}$ is weakly convex, then $J_{a}^{-1}$ is weakly concave. By Jensen's inequality and the law of iterated expectations, the expected payment is higher for $d$ than for $d^{\prime}$ because the critical virtual valuation for the earlier deadline is less variable. If $J_{a}$ is concave, we get the opposite observation. The following theorem generalizes the insight to all valuations $v \in[0, \bar{v}]$. For the case of $v<\bar{v}$, the expected payoff also depends on the winning probability which requires a different proof which uses second-order stochastic dominance.

Theorem 3. (i) If $J_{a}(v)$ is weakly convex for all a, then $\left(x^{r l x}, y^{r l x}\right)$ is incentive compatible and maximizes the seller's revenue if both the valuation and the deadline are private information.

(ii) If $J_{a}(v)$ is strictly concave for some a, then $\left(x^{r l x}, y^{r l x}\right)$ is not incentive compatible if the deadline and the valuation are private information. 
Proof. The proof can be found in Appendix A.

There are two important elements in this payment rule that drive Theorem 3. First, the arrival of new information over time leads to in creased dispersion in the critical virtual valuation. Second, the seller's desire to minimize information rents for the valuation leads to a markup that is typically non-linear. These two elements, arrival of information, and the desire to extract information rents interact, and distort the incentives to report the deadline truthfully. If the seller maximized value instead of revenue, the markup would disappear and the relaxed solution would always be incentive compatible. ${ }^{26}$ Moreover, if there was no information revelation over time, but the seller maximized revenue, the relaxed solution would again be always incentive compatible. ${ }^{27}$

Remark 1 (Ex-Post Incentive Compatibility). Note that in Lemma 2, strict second-order stochastic dominance is shown conditional on the state in the arrival period. This implies that the incentive compatibility result of Theorem 3.i also holds if buyers can condition their reports on the state at their arrival time. In other words, under the conditions of Theorem 3.i, the relaxed solution is periodic ex-post incentive compatible. ${ }^{28}$ This shows that the optimal solution does not rely on the seller's ability to conceal information.

Remark 2 (Convex and Concave Virtual Valuations). In static mechanism design, the regularity condition that guarantees incentive compatibility of the relaxed solution (Assumption 1) is satisfied by a large class of distributions (see Ewerhart, 2013). In the present model, the picture is less clear. Strict concavity (weak convexity) of the virtual valuation is equivalent to

$$
\frac{1-F(v)}{(f(v))^{2}}\left(f(v) f^{\prime \prime}(v)-2\left(f^{\prime}(v)\right)^{2}\right) \stackrel{(\gtrless)}{<} f^{\prime}(v) .
$$

This implies that all distributions with an increasing density that is not too convex have strictly concave virtual valuations. Conversely, decreasing densities that are not too concave imply weak convexity of the virtual valuation. For example, the virtual valuation is concave if the density is linear and increasing $(f(v)=1-k+2 k v, k \in(0,1])$, or a power of $v\left(f(v)=(k+1) v^{k}, k>0\right)$, but we also have concavity for hump- and U-shaped densities $\left(f(v)=\frac{3}{2}-6\left(v-\frac{1}{2}\right)^{2}\right.$ and $\left.f(v)=12\left(v-\frac{1}{2}\right)^{2}\right)$. The virtual valuation is linear or convex if the density is linear and decreasing $\left(f(v)=(k+1) v^{k}, k \leq 0\right)$ or a power of $1-v\left(f(v)=(1+k)(1-v)^{k}\right)$. These examples show clearly that both the regular and the irregular case are economically relevant. We will therefore study the optimal mechanism in the irregular case in Section 4.

\footnotetext{
${ }^{26}$ This also follows from general existence results for efficient dynamic mechanisms (Parkes and Singh, 2003; Bergemann and Välimäki, 2010).

${ }^{27}$ In this (hypothetical) case, the seller has perfect foresight and we are essentially back in the static model of Myerson (1981).

${ }^{28}$ This hybrid concept requires ex-post incentive compatibility with respect to all information that is realized in the current period and Bayesian incentive compatibility for all information that realizes in the future (see Bergemann and Välimäki, 2010).
} 
3.4. Generalizations. The martingale property of $\underline{J}_{a}\left(H_{d}^{-i}\right)$ does not depend on the assumption that the valuation and the deadline are conditionally independent. Appendix C.2 in the Supplemental Material shows the martingale property for the case of two periods $(T=2)$, a finite number of units $(K>1)$ and buyers with unit demand. We can therefore generalize Theorem 3 to allow for correlations and multiple units. ${ }^{29}$

For the case that the valuation and the deadline of a buyer are not independent, the sufficient conditions in Theorem 3 have to be augmented. If the distribution of the valuation becomes weaker for later deadlines (in the hazard-rate order), then the seller will set prices less aggressively if a buyer has a later deadline. This makes it more likely that the relaxed solution is incentive compatible if the deadline is private. Conversely, if the distribution of valuations becomes stronger for later deadlines, the markups used in the relaxed solution increase with the deadline, which makes it less likely that the relaxed solution is incentive compatible. $^{30}$ Combining the hazard-rate order with the previous convexity/concavity conditions, we the following sufficient conditions for incentive compatibility of the relaxed solution.

Theorem (3'). Suppose that $K=1$ and $T<\infty$, or that $T=2$ and $K<\infty$. Suppose that both the valuation and the deadline are private information. Then $\left(x^{r l x}, y^{r l x}\right)$

(i) is incentive compatible and maximizes the seller's revenue if for all $a \leq d<d^{\prime} \leq T$

(a) $J_{a}(v \mid d) \leq J_{a}\left(v \mid d^{\prime}\right)$ for all $v \in[0, \bar{v}]$, and

(b) $J_{a}(v \mid d)$ or $J_{a}\left(v \mid d^{\prime}\right)$ is weakly convex as a function of $v$.

(ii) violates $\left(\mathrm{ICD}^{\mathrm{d}}\right)$ if for some $a \leq d<d^{\prime} \leq T$

(a) $J_{a}(v \mid d) \geq J_{a}\left(v \mid d^{\prime}\right)$ for all $v \in[0, \bar{v}]$, and

(b) $J_{a}(v \mid d)$ or $J_{a}\left(v \mid d^{\prime}\right)$ is strictly concave as a function of $v$.

\section{The General Solution}

In this section, we analyze how a binding incentive constraint for the deadline distorts the optimal allocation rule. The most important difference in comparison to the relaxed solution is that the binding incentive constraint leads to bunching of deadlines.

In the irregular case, the analysis of the seller's problem is significantly more complex. We obtain an explicit solution for the case of two periods $(T=2)$ with deterministic arrival of one buyer in each period. We will assume that the profit of a monopolist who is selling to the first buyer is concave, which is captured by Assumption 2. This assumption ensures that the optimal mechanism does not use lotteries in the first period. While Assumption 2 is needed for a complete solution, a main property of the optimal solution, namely that deadlines are not separated for high valuations, is robust.

\footnotetext{
${ }^{29}$ I conjecture that Lemma 2 generalizes to the case of many objects (with unit demand) and more than two time periods. This would imply an immediate generalization of Theorem 3'. The main obstacle for a proof of the conjecture is that the formulation of the optimal allocation rule in terms of a score is not easily generalizable beyond the case of one object.

${ }^{30}$ The observation is not new. It can also be found in the previous literature on static models with two-dimensional private information. See Section 1.1 and Footnote 7.
} 
For the $2 \times 2$ model we simplify notation. The deadline of the first buyer is denoted by $d \in\{1,2\}$, and the probability of $d=1$ by $\rho . x_{1}\left(v_{1}, d=1\right)$ denotes the winning probability of buyer if his deadline is one, and $x_{i}\left(v_{1}, d, v_{2}\right)$ denotes the conditional winning probability of buyer $i$ in period two, for a given type profile, conditional on the event that the object has not been allocated in the first period. Note that an allocation rule $x$ is feasible if and only if for all $v_{1}, v_{2} \in[0, \bar{v}], d \in\{1,2\}$, and $i \in\{1,2\}: x_{1}\left(v_{1}, 1\right), x_{i}\left(v_{1}, d, v_{2}\right) \in[0,1]$ and $^{31}$

$$
x_{1}\left(v_{1}, 2, v_{2}\right)+x_{2}\left(v_{1}, 2, v_{2}\right) \leq 1 .
$$

We write interim winning probabilities of buyer one as

$$
q_{1}\left(v_{1}, 1\right)=x_{1}\left(v_{1}, 1\right), \quad \text { and } \quad q_{1}\left(v_{1}, 2\right)=\int_{0}^{\bar{v}} x_{1}\left(v_{1}, 2, v_{2}\right) f_{2}\left(v_{2}\right) d v_{2} .
$$

The interim winning probability of buyer two, conditional on the deadline of buyer one, and the event that the object has not been allocated in period one, is given by ${ }^{32}$

$$
\begin{aligned}
q_{2}\left(v_{2} \mid d=1\right) & :=\int_{0}^{\bar{v}} x_{2}\left(v_{1}, 1, v_{2}\right) \frac{\left(1-x_{1}\left(v_{1}, 1\right)\right) f_{1}\left(v_{1}\right)}{\int_{0}^{\bar{v}}\left(1-x_{1}(s, 1)\right) f_{1}(s) d s} d v_{1}, \\
\text { and } \quad q_{2}\left(v_{2} \mid d=2\right) & :=\int_{0}^{\bar{v}} x_{2}\left(v_{1}, 2, v_{2}\right) f_{1}\left(v_{1}\right) d v_{1} .
\end{aligned}
$$

With this notation, the seller's objective can be written as follows:

$$
\rho \pi_{1}\left[q_{1}(\cdot, 1), q_{2}(\cdot \mid 1)\right]+(1-\rho) \pi_{2}\left[q_{1}(\cdot, 2), q_{2}(\cdot \mid 2)\right] .
$$

The first part of the objective, $\pi_{1}$, is the expected profit conditional on $d=1$, which does not depend on the allocation rule for $d=2$ :

$\pi_{1}\left[q_{1}(\cdot, 1), q_{2}(\cdot \mid 1)\right]=\int_{0}^{\bar{v}}\left[q_{1}\left(v_{1}, 1\right) J_{1}\left(v_{1}\right)+\left(1-q_{1}\left(v_{1}, 1\right)\right) \int_{0}^{\bar{v}} q_{2}\left(v_{2} \mid 1\right) J_{2}\left(v_{2}\right) f_{2}\left(v_{2}\right) d v_{2}\right] f_{1}\left(v_{1}\right) d v_{1}$.

Similarly, $\pi_{2}$, the expected profit conditional on $d=2$, does not depend on the allocation rule for $d=1$ :

$$
\pi_{2}\left[q_{1}(\cdot, 2), q_{2}(\cdot \mid 2)\right]=\int_{0}^{\bar{v}} q_{1}(v, 2) J_{1}(v) f_{1}(v)+q_{2}(v \mid 2) J_{2}(v) f_{2}(v) d v .
$$

The seller maximizes (4.3) subject to the constraint that $q$ is the reduced form of a feasible allocation rule and incentive constraints: For all $d \in\{1,2\}$, and all $v, v^{\prime} \in[0, \bar{v}]$ :

$$
\begin{aligned}
v & >v^{\prime} \Rightarrow q_{1}(v, d) \geq q_{1}\left(v^{\prime}, d\right), \\
\text { and } \quad U_{1}(v, 1) & =\int_{0}^{v} q_{1}(s, 1) d s \leq \int_{0}^{v} q_{1}(s, 2) d s=U_{1}(v, 2)
\end{aligned}
$$

Given that virtual valuations are assumed to be increasing, the monotonicity constraint on $q_{2}$ can be relaxed and is therefore omitted. As before, the seller maximizes virtual

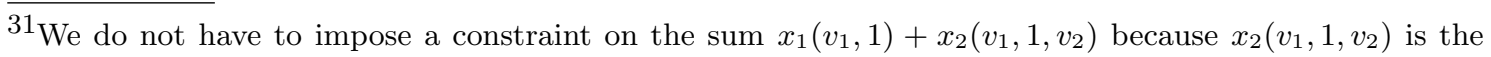
winning probability of buyer two conditional on the event that the object has not been allocated in the first period.

${ }^{32}$ With these definitions, we have $q_{2}\left(v_{2}\right)=\rho\left(\int_{0}^{\bar{v}}\left(1-x_{1}\left(v_{1}, 1\right)\right) f_{1}\left(v_{1}\right) d v_{1}\right) q_{2}\left(v_{2} \mid d=1\right)+(1-\rho) q_{2}\left(v_{2} \mid d=2\right)$.
} 
surplus. In contrast to the previous section, however, the incentive constraint for the deadline $\left(\mathrm{ICD}_{1}^{\mathrm{d}}\right)$ is imposed on the seller's problem. Note that this is the only constraint that involves the allocation rule for both $d=1$ and $d=2$. Therefore, we separate the two sub-problems in the following. We will first derive the optimal allocation rule for $d=1$, taking $U_{1}(\cdot, 2)$ as given. Second, we will derive the optimal allocation rule for $d=2$, taking $U_{1}(\cdot, 1)$-which is the type-dependent participation constraint of buyer one, as given. These steps will reveal the distortions that the seller uses in the first and second period, respectively, in response to the binding incentive constraint. Finally, we will combine the two results to obtain an optimal allocation rule for the complete problem.

4.1. The Optimal Allocation conditional on $d=1$. If buyer one is impatient $(d=1)$, the optimal allocation rule is determined recursively. If the object has not been sold in period one, it is clear from (4.4) that it is optimal to sell to buyer two if and only if $J_{2}\left(v_{2}\right) \geq 0$. Hence, we have

$$
q_{2}^{*}\left(v_{2} \mid 1\right)= \begin{cases}0, & \text { if } J_{2}\left(v_{2}\right)<0, \\ 1, & \text { otherwise, }\end{cases}
$$

and the option value of waiting for the second period is $V^{\mathrm{opt}}:=\int_{0}^{\bar{v}} q_{2}^{*}\left(v_{2} \mid 1\right) J_{2}\left(v_{2}\right) f_{2}\left(v_{2}\right) d v_{2}$. Inserting this into (4.4), we can write the problem of maximizing $\pi_{1}$ for given $U_{1}^{*}(\cdot, 2)$ as the problem $\mathcal{P}_{1}$ :

$$
\begin{gathered}
\max _{q_{1}\left(v_{1}, 1\right)} \int_{0}^{\bar{v}}\left[q_{1}\left(v_{1}, 1\right) J_{1}\left(v_{1}\right)+\left(1-q_{1}\left(v_{1}, 1\right)\right) V^{\mathrm{opt}}\right] f_{1}\left(v_{1}\right) d v_{1} \\
\text { s.t. } \quad q_{1}(v, 1) \in[0,1],\left(M_{1}\right), \\
\text { and } \forall v_{1} \in[0, \bar{v}]: \quad U_{1}\left(v_{1}, 1\right)=\int_{0}^{v_{1}} q_{1}(s, 1) d s \leq U_{1}^{*}\left(v_{1}, 2\right),
\end{gathered}
$$

where $U_{1}^{*}(\cdot, 2)$ is convex, and satisfies $\partial U_{1}^{*}\left(v_{1}, 2\right) / \partial v_{1} \in[0,1]$ and $U_{1}^{*}(0,2)=0$.

If (4.6) is ignored, the optimal solution for $q_{1}\left(v_{1}, 1\right)$ is given by a cutoff rule: $q_{1}\left(v_{1}, 1\right)=1$ if buyer one's virtual valuation exceeds $V^{\text {opt }}$ and $q_{1}\left(v_{1}, 1\right)=0$ otherwise. Therefore, conditional on $d=1$, the optimal solution can be implemented by a sequence of fixed prices. The price $r_{1}$ posted in the first period is given by $r_{1}=J_{1}^{-1}\left(V^{\mathrm{opt}}\right)$, and the price in the second period $r_{2}$ is given by $r_{2}=J_{2}^{-1}(0)$. Conditional on the types of the buyers, the allocation decision is deterministic. This is the well-known no-haggling result of Riley and Zeckhauser (1983).

The following lemma shows that if $q_{1}$ is a cutoff rule, it suffices to check (4.6) for $v_{1}=\bar{v}$.

Lemma 3. If $q_{1}\left(v_{1}, 1\right) \in\{0,1\}$ for all $v_{1} \in[0, \bar{v}]$ and satisfies $\left(\mathrm{M}_{1}\right)$, then $\left(\mathrm{ICD}_{1}^{\mathrm{d}}\right)$ holds for any $v$, if it is fulfilled for $v=0$ and $v=\bar{v}$.

Proof. $q_{1}(v, 1)$ jumps from zero to one at $v=\bar{v}-U_{1}(\bar{v}, 1)$ if the allocation is monotone and $q_{1}\left(v_{1}, 1\right) \in\{0,1\}$. Therefore, the expected utility for $d=1$ is constant on $\left[0, \bar{v}-U_{1}(\bar{v}, 1)\right]$ and has slope one on $\left[\bar{v}-U_{1}(\bar{v}, 1), \bar{v}\right]$. Since $d U_{1}(v, 2) / d v \in[0,1], U_{1}(v, 2)$ cannot get "too convex", and cannot cross $U_{1}(v, 1)$ from below if $U_{1}(0,2) \geq U_{1}(0,1)$. Therefore, we 
must have $U_{1}(v, 2) \geq U_{1}(v, 1)$ for all $v \in[0, \bar{v}]$ if this constraint is fulfilled for $v=0$ and $v=\bar{v}$

This Lemma implies that the types for which the constraint is binding are independent of the allocation rule, as long as the seller uses a cutoff rule in the first period. In particular, since $U_{1}(0,1)=U_{2}(0,2)=0$ the incentive constraint for the deadline is reduced to a single inequality. Unfortunately, the no-haggling result of Riley and Zeckhauser (1983) need not hold if we impose (4.6). ${ }^{33}$ The following theorem shows, however, that under Assumption $2, q_{1}^{*}\left(v_{1}, 1\right)$ is always a cutoff rule. To simplify notation we write $\bar{U}=U_{1}^{*}(\bar{v}, 2)$. With slight abuse of notation, we also use $\pi_{1}(\bar{U})$ to denote the value of $\mathcal{P}_{1}$ if (4.6) is imposed only for $v_{1}=\bar{v}$.

Theorem 4. Suppose $f_{1}$ satisfies Assumption 2, Then

(i) the optimal solution of $\mathcal{P}_{1}$ is deterministic, it only depends on $\bar{U}$, and is given by

$$
q_{1}^{*}\left(v_{1}, 1\right)= \begin{cases}0, & \text { if } J_{1}\left(v_{1}\right)<\max \left\{V_{2}^{\mathrm{opt}}, J_{1}(\bar{v}-\bar{U})\right\}, \\ 1, & \text { otherwise, }\end{cases}
$$

(ii) $\pi_{1}(\bar{U})$ is continuously differentiable and concave for $\bar{U} \in(0, \bar{v})$ and strictly concave if $\bar{U}<\bar{v}-J_{1}^{-1}\left(V^{o p t}\right)$.

Proof. The proof of (i) uses a simple variational argument. In (4.6), winning probabilities are not weighted in the integral because incentive compatibility constraints are independent of the buyer's own distribution function. In the objective, however, $q_{1}\left(v_{1}, 1\right)$ is weighted by $\left(J_{1}\left(v_{1}\right)-V_{2}^{\text {opt }}\right) f_{1}\left(v_{1}\right)$. Increasing the winning probability $q_{1}\left(v_{1}, 1\right)$ for valuations in $[v, v+\varepsilon]$, and decreasing it by the same amount on $\left[v^{\prime}, v^{\prime}+\varepsilon\right]$, with $v^{\prime}+\varepsilon \leq v$, decreases $U_{1}\left(v_{1}, 1\right)$ for $v_{1} \in\left[v^{\prime}, v+\varepsilon\right]$ and leaves $U_{1}\left(v_{1}, 1\right)$ unchanged otherwise. Hence, such a change in $q_{1}$ does not destroy incentive compatibility. On the other hand, this shift of winning probability from low to high types increases the seller's revenue if $\left(J_{1}\left(v_{1}\right)-V_{2}^{\text {opt }}\right) f_{1}\left(v_{1}\right)$ is increasing. Assumption 2 guarantees that $\left(J_{1}\left(v_{1}\right)-V_{2}^{\mathrm{opt}}\right) f_{1}\left(v_{1}\right)$ is increasing whenever $J_{1}\left(v_{1}\right)-V_{2}^{\text {opt }} \geq 0$. Therefore, the winning probability must jump from zero to one at some point $r_{1}$ and the allocation is deterministic. $r_{1}$ is the lowest value such that $J_{1}\left(r_{1}\right) \geq V_{2}^{\text {opt }}$ and $U_{1}(\bar{v}, 1)=\bar{v}-r_{1} \leq U(\bar{v})$. This yields $r_{1}=\max \left\{J_{1}^{-1}\left(V_{2}^{\mathrm{opt}}\right), \bar{v}-\bar{U}\right\}$.

If we insert the optimal solution in the objective function we obtain

$$
\begin{aligned}
& \pi_{1}(\bar{U})=\int_{r_{1}}^{\bar{v}} J_{1}(v) f_{1}(v) d v+V_{2}^{\mathrm{opt}} F_{1}\left(r_{1}\right) . \\
& \pi_{1}^{\prime}(\bar{U})= \begin{cases}\left(J_{1}(\bar{v}-\bar{U})-V_{2}^{\mathrm{opt}}\right) f_{1}(\bar{v}-\bar{U}), & \text { if } J_{1}(\bar{v}-\bar{U})>V_{2}^{\mathrm{opt}}, \\
0 & \text { otherwise. }\end{cases}
\end{aligned}
$$

\footnotetext{
${ }^{33}$ Riley and Zeckhauser's result is a consequence of the structure of the feasible set of the maximization problem. Manelli and Vincent (2007) show that the set of extremal points of the feasible set, which contains the maximizers, is equal to the set of deterministic allocation rules. Due to the additional constraint (3), the set of extremal points changes. Rather than trying to extend the results of Manelli and Vincent here, we use Assumption 2 as a sufficient condition for a deterministic mechanism.
} 
For $\bar{U} \rightarrow \bar{v}-J_{1}^{-1}\left(V_{2}^{\text {opt }}\right)$ we have $\pi_{1}^{\prime}(\bar{U}) \rightarrow 0$ since $f_{1}$ is bounded. Hence, $\pi_{1}^{\prime}(\bar{U})$ is continuous. Using Assumption 2, we conclude that $\pi_{1}^{\prime}(\bar{U})$ is strictly decreasing if $J_{1}(\bar{v}-\bar{U})>V_{2}^{\text {opt }}$ and hence $\pi_{1}$ is strictly concave.

If Assumption 2 does not hold, raising the winning probability for a lower valuation may be more profitable than for a higher valuation because $f_{1}(v)$ is decreasing sharply and the higher likelihood of a low type outweighs the lower virtual valuation.

Distortions if Buyer 1 is Impatient. Theorem 4 shows that the optimal allocation rule for $d=1$ allocates less often to buyer one than the relaxed solution. If $J_{1}(\bar{U}-\bar{v})>V_{2}^{\text {opt }}$, the optimal posted price in period one equals $\bar{U}-\bar{v}$. This price no longer reflects the opportunity cost of the seller plus a mark-up. Instead, it is entirely determined by the binding incentive constraint for the deadline. It is distorted upwards compared to the relaxed solution, to make the fixed price less attractive for a patient buyer.

4.2. The Optimal Allocation conditional on $d=2$. In the previous section, we have shown that the optimal allocation rule $q_{1}^{*}\left(v_{1}, 1\right)$ is a cutoff rule if Assumption 2 is satisfied. Therefore, we impose $\left(\operatorname{ICD}_{1}^{\mathrm{d}}\right)$ only for $v_{1}=\bar{v}$ in the analysis if the optimal allocation conditional on $d=2$. Setting $\bar{U}:=\int_{0}^{\bar{v}} q_{1}^{*}\left(v_{1}, 1\right) d v_{1}$, we obtain problem $\mathcal{P}_{2}$ which yields the optimal allocation rule for $d=2$ :

$$
\begin{aligned}
\pi_{2}(\bar{U}):= & \max _{q} \int_{0}^{\bar{v}} q_{1}(v, 2) J_{1}(v) f_{1}(v)+q_{2}(v \mid 2) J_{2}(v) f_{2}(v) d v \\
& \text { s.t. }(\mathrm{F}),\left(\mathrm{M}_{1}\right), \\
& \text { and } \int_{0}^{\bar{v}} q_{1}(v, 2) d v \geq \bar{U} .
\end{aligned}
$$

In Appendix B, we show that $(\mathrm{F})$ can be used to eliminate $q_{2}(v \mid 2)$ and transform this maximization problem into a solvable optimal control problem. Here we give an informal argument to derive the optimal solution. If we ignore the monotonicity constraint $\left(\mathrm{M}_{1}\right)$ but maintain the constraint (F), we can use the following Lagrangian relaxation to get an intuition for the optimal solution:

$$
\mathcal{L}=\int_{0}^{\bar{v}} q_{1}(v, 2) J_{1}(v) f_{1}(v)+q_{2}(v \mid 2) J_{2}(v) f_{2}(v) d v-p_{U}\left[\bar{U}-\int_{0}^{\bar{v}} q_{1}(v, 2) d v\right]
$$

Rearranging the Lagrangian, and inserting (4.1) and (4.2), we obtain the objective ${ }^{34}$

$$
\mathcal{L}=\int_{0}^{\bar{v}} \int_{0}^{\bar{v}}\left[x_{1}\left(v_{1}, 2, v_{2}\right) J_{1}^{p_{U}}\left(v_{1}\right)+x_{2}\left(v_{1}, 2, v_{2}\right) J_{2}\left(v_{2}\right)\right] f_{1}(v) f_{2}(v) d v_{1} d v_{2},
$$

where $J_{1}^{p_{U}}(v):=J_{1}(v)+\frac{p_{U}}{f_{1}(v)}$.

Now that the seller's objective is formulated in terms of the allocation rule $x$ rather than the reduced form, we can apply the feasibility constraint $(\mathrm{F})$ directly. If we know

\footnotetext{
$\overline{34}$ If the participation constraint is imposed for all valuations, we obtain a similar formulation with a type dependent multiplier $p_{U}(v)$ and generalized virtual valuation $\tilde{J}_{1}(v)=J_{1}(v)+\frac{p_{U}(v)}{f(v)}$.
} 
the value of the Lagrange multiplier $p_{U}$, then the optimal allocation rule is obtained by point-wise maximization

$$
\begin{aligned}
& x_{1}\left(v_{1}, 2, v_{2}\right)= \begin{cases}0, & \text { if } J_{1}^{p_{U}}\left(v_{1}\right)<\max \left\{0, J_{2}\left(v_{2}\right)\right\} \\
1, & \text { otherwise },\end{cases} \\
& x_{2}\left(v_{1}, 2, v_{2}\right)= \begin{cases}0, & \text { if } J_{2}\left(v_{2}\right) \leq \max \left\{0, J_{1}^{p_{U}}\left(v_{1}\right)\right\}, \\
1, & \text { otherwise. }\end{cases}
\end{aligned}
$$

In this allocation rule, the virtual valuation $J_{1}$ has been replaced by $J_{1}^{p_{U}}$, which we call the generalized virtual valuation. With the standard virtual valuation (i.e., if $p_{U}=0$ ), the optimal allocation rule is monotonic if Assumption 1 is fulfilled. Here, however, we have to ensure that $J_{1}^{p_{U}}$ is non-decreasing. Since $J_{1}^{p_{U}}$ depends on the endogenous multiplier $p_{U}$, this is difficult to establish unless we impose strong conditions on the type distribution. ${ }^{35}$ Therefore, we show that the optimal allocation rule subject to $\left(\mathrm{M}_{1}\right)$ can be obtained by ironing the generalized virtual valuation.

Definition 3 (Ironing; Myerson, 1981). For every $v_{1} \in[0, \bar{v}]$, and every $p_{U} \geq 0$, define the ironed generalized virtual valuation by ${ }^{36}$

$$
\bar{J}_{1}^{p_{U}}\left(v_{1}\right):=\left.\frac{d}{d t}\left[\operatorname{conv}\left(\int_{0}^{t} J_{1}\left(F_{1}^{-1}(s)\right)+\frac{p_{U}}{f_{1}\left(F_{1}^{-1}(s)\right)} d s\right)\right]\right|_{t=F_{1}\left(v_{1}\right)} .
$$

Using Definition (3), we obtain a monotonic virtual valuation that allows to describe the optimal allocation rule by replacing $J_{1}^{p_{U}}\left(v_{1}\right)$ by $\bar{J}_{1}^{p_{U}}\left(v_{1}\right)$ in (4.8). With this modification, it may be the case that $\bar{J}_{1}^{p_{U}}\left(v_{1}\right)$ equals zero for an interval of valuations $v_{1}$. Since a tie can therefore occur with positive probability (i.e. whenever $J_{2}\left(v_{2}\right)<0$ and $\bar{J}_{1}^{p_{U}}\left(v_{1}\right)=0$ ), the tie-breaking rule has to be specified explicitly. To do so, we extend the definition of the allocation rule by an additional parameter $\underline{x}_{1}^{0}$, which specifies the winning probability of buyer one if $\bar{J}_{1}^{p_{U}}\left(v_{1}\right)=0$ and $J_{2}\left(v_{2}\right)<0$. For given $p_{U}$ and $\underline{x}_{1}^{0}$ the optimal allocation

\footnotetext{
$\overline{35}$ For example, a sufficient condition is that $f_{1}(v)$ is non-increasing.

${ }^{36}$ This definition uses the following steps taken from Myerson (1981): First, for all $t \in[0,1]$, we define $M_{1}^{p_{U}}(t):=J_{1}\left(F_{1}^{-1}(t)\right)+\frac{p_{U}}{f_{1}\left(F_{1}^{-1}(t)\right)}$ as the generalized virtual valuation at the $t$-quantile of $F_{1}$. Second, we integrate this function: $\mathcal{M}^{p_{U}}(t):=\int_{0}^{t} M_{1}^{p_{U}}(s) d s$. Then we take the convex hull (that is, the greatest convex function $G$ such that $G(t) \leq \mathcal{M}^{p_{U}}(t)$ for all $\left.t\right): \overline{\mathcal{M}}^{p_{U}}(t):=\operatorname{conv} \mathcal{M}^{p_{U}}(t)$. Since $\overline{\mathcal{M}}^{p_{U}}$ is convex, it is almost everywhere differentiable, any selection $\bar{M}_{1}^{p_{U}}(t)$ from the sub-gradient is non-decreasing and the selection is unique almost everywhere. Finally we reverse the change of variables made in the first step to obtain the ironed generalized virtual valuation $\bar{J}_{1}^{p_{U}}\left(v_{1}\right):=\bar{M}_{1}^{p_{U}}\left(F_{1}\left(v_{1}\right)\right)$. See Reid (1968) for a similar construction in an optimal control problem.
} 
rule then is given by

$$
\begin{aligned}
& \bar{x}_{1}\left(v_{1}, 2, v_{2}\right)= \begin{cases}1, & \text { if } \bar{J}_{1}^{p_{U}}\left(v_{1}\right)>0 \text { and } \bar{J}_{1}^{p_{U}}\left(v_{1}\right) \geq J_{2}\left(v_{2}\right) \\
\underline{x}_{1}^{0}, & \text { if } \bar{J}_{1}^{p_{U}}\left(v_{1}\right)=0 \text { and } J_{2}\left(v_{2}\right) \leq 0, \\
0, & \text { otherwise },\end{cases} \\
& \bar{x}_{2}\left(v_{1}, 2, v_{2}\right)= \begin{cases}0, & \text { if } J_{2}\left(v_{2}\right) \leq \max \left\{0, \bar{J}_{1}^{p_{U}}\left(v_{1}\right)\right\}, \\
1, & \text { otherwise. }\end{cases}
\end{aligned}
$$

It remains to compute the multiplier $p_{U}$ and the parameter $\underline{x}_{1}^{0}$ such that $U_{1}(\bar{v}, 2)=$ $\int_{0}^{\bar{v}} \bar{q}_{1}(v, 2) d v=\bar{U}$. The following lemma shows that there is a unique such pair. (If $U_{1}(\bar{v}, 2)>\bar{U}$ for $p_{U}=0$, the constraint is not binding at the optimal solution and we must have $p_{U}=\underline{x}_{1}^{0}=0$.)

Lemma 4. Let $\bar{U} \in[0, \bar{v}]$ such that $\int_{0}^{\bar{v}} \bar{q}_{1}(v, 2) d v<\bar{U}$ if we set $p_{U}=0$ and $\underline{x}_{1}^{0}=0$ in (4.9). Then there exists a unique multiplier $p_{U} \geq 0$ and a unique probability $\underline{x}_{1}^{0} \in[0,1]$, such that the solution defined by (4.9) satisfies $\int_{0}^{\bar{v}} \bar{q}_{1}(v, 2) d v=\bar{U}$. They are given by

$$
\begin{aligned}
& p_{\bar{U}}^{*}:=\min \left\{p_{U} \geq 0 \mid \int_{0}^{\bar{v}} \bar{q}_{1}(v, 2) d v \geq \bar{U} \text { if } \underline{x}_{1}^{0}=1\right\}, \\
& \underline{x}_{1}^{0 *}:=\min \left\{\underline{x}_{1}^{0} \geq 0 \mid \int_{0}^{\bar{v}} \bar{q}_{1}(v, 2) d v \geq \bar{U} \text { if } p_{\bar{U}}=p_{\bar{U}}^{*}\right\} .
\end{aligned}
$$

Proof. Let $\mathcal{U}\left(p_{U}\right)$ be the set of possible values of $U_{1}(\bar{v}, 2)=\int_{0}^{\bar{v}} \bar{q}_{1}(v, 2) d v$ that can be achieved by setting $\underline{x}_{1}^{0} \in[0,1]$. For $p_{U}$ sufficiently high, $\bar{J}_{1}^{p_{U}}\left(v_{1}\right)>\bar{v}$ for all $v_{1} \in[0, \bar{v}]$, hence there exists a value $\bar{p}_{U}$ such that $\int_{0}^{\bar{v}} \bar{q}_{1}(v, 2) d v=\bar{v}$ so that $\bar{v} \in \mathcal{U}\left(\bar{p}_{U}\right)$. In Appendix $\mathrm{B}$, we show that $\mathcal{U}\left(p_{U}\right)$ is an increasing, closed-valued, and upper-hemicontinuous correspondence. Therefore we can find the desired $p_{U}$ and $\underline{x}_{1}^{0} \in[0,1]$ for all $\bar{U} \in[0, \bar{v}]$. Uniqueness follows from the fact that $\mathcal{U}\left(p_{U}\right)$ is increasing.

The following theorem shows that the optimal allocation rule that we have derived informally, is indeed an optimal solution to $\mathcal{P}_{2}$.

Theorem 5. (i) The reduced form of (4.9) for $p_{\bar{U}}^{*}$ and $\underline{x}_{1}^{0 *}$ is an optimal solution of $\mathcal{P}_{2}$.

(ii) Fix $\bar{U}$ and suppose that $J_{1}^{p_{U}^{*}}\left(v_{1}\right)$ is strictly increasing in $v_{1}$. Then the reduced form of (4.8) for $p_{U}=p_{\bar{U}}^{*}$ is an optimal solution of $\mathcal{P}_{2}$.

(iii) For almost every $\bar{U} \leq \bar{v}, \pi_{2}^{\prime}(\bar{U})=-p_{U}^{*}$.

(iv) $\pi_{2}(\bar{U})$ is weakly concave if $p_{\bar{U}}^{*}>0$ and strictly concave if $\bar{J}_{1}^{p_{U}}\left(v_{1}\right)=0$ has a unique solution.

Proof. See Appendix B.

Distortions if Buyer 1 is Patient. We see that the optimal allocation rule conditional on $d=2$ involves a "bonus" for buyer one. Even if the valuations of both buyers are identically distributed $\left(f_{1}=f_{2}\right)$, buyer one has a higher chance of winning than buyer two 


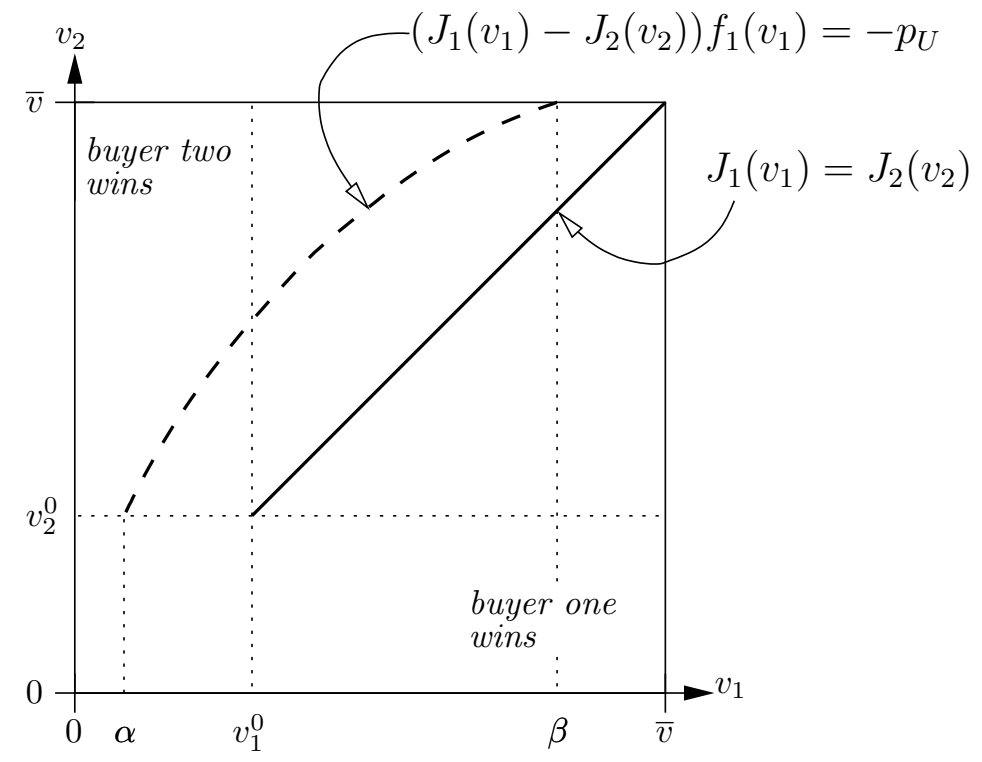

Figure 4.1. Relaxed solution: valuations tie at the solid line. General solution: valuations tie at the dashed line.

(for a given valuation) because

$$
\forall v_{1} \in[0, \bar{v}]: \quad J_{1}^{p_{U}}\left(v_{1}\right)=J_{1}\left(v_{1}\right)+\frac{p_{U}}{f_{1}\left(v_{1}\right)}>J_{1}\left(v_{1}\right),
$$

if the relaxed solution is not incentive compatible $\left(p_{U}>0\right)$. See Figure 4.1 for an illustration. This is leads to a lower reserve price $\alpha<v_{1}^{0}$ for buyer one, which increases the efficiency of the allocation compared to the relaxed solution. Overall, the efficiency comparison is nevertheless ambiguous because buyer one is also given an advantage over buyer two if the latter has a positive virtual valuation. If valuations are identically distributed, this leads to a loss in efficiency compared to the relaxed solution, but if buyer one has a sufficiently strong type distribution, the overall efficiency effect of the distortion can be positive.

Remarkably, whenever the incentive constraint for the deadline is binding $\left(p_{U}>0\right)$, there is a non-empty interval $(\beta, \bar{v}]$, such that $J_{1}^{p_{U}}\left(v_{1}\right)>\bar{v}$ for all valuations $v_{1} \in(\beta, \bar{v}]$. Buyer two cannot win against buyer one if $v_{1}>\beta$, and buyer one has a winning probability of one even if he does not have the highest possible valuation. The familiar result that there is no distortion at the top does not hold here.

4.3. Complete Solution. The final step in the analysis of the seller's problem is to determine $\bar{U}$. The expected profit of the seller is given by $\rho \pi_{1}(\bar{U})+(1-\rho) \pi_{2}(\bar{U})$, where $\bar{U}$ is the designer's choice which determines the magnitude of the distortions in the two sub-problems for $d=1,2$. Depending on the chosen value of $\bar{U}$, the constraints (4.6) and (4.7) may be binding or not. Given that both $\pi_{1}$ and $\pi_{2}$ are concave, the optimal value of 
$\bar{U}$ is determined by the first-order condition

$$
\rho \pi_{1}^{\prime}(\bar{U})=-(1-\rho) \pi_{2}^{\prime}(\bar{U}) .
$$

Using that $-\pi_{2}^{\prime}(\bar{U})=p_{U}$, we obtain that the optimal solution is given by

$$
\begin{aligned}
p_{U} & =\frac{\rho}{1-\rho} \pi_{1}^{\prime}(\bar{U}), \\
\text { and } \quad \bar{U} & \leq \int_{0}^{\bar{v}} \bar{q}_{1}^{p_{U}}(v, 2) d v_{1}, \quad \text { with equality if } p_{U}>0,
\end{aligned}
$$

where $\bar{q}^{p_{U}}$ is the reduced form of (4.9) for given value of $p_{U}$. An explicit expression for $\bar{U}$ or $p_{U}$ is not available. For given $p_{U}$, however, $U_{1}(\bar{v}, 2)=\int_{0}^{\bar{v}} \bar{q}_{1}^{p_{U}}(v, 2) d v_{1}$ is easy to calculate numerically and an explicit expression for $\pi_{1}^{\prime}$ is given in the proof of Theorem 4 . Hence, it is easy to compute the optimal values of $p_{\bar{U}}$ and $\bar{U}$ numerically.

To conclude the derivation of the optimal mechanism, we note that $q_{2}\left(v_{2} \mid 1\right)$, defined by the fixed price $r_{2}$, and $q_{2}\left(v_{2} \mid 2\right)$, defined by the reduced form of (4.9), are non-decreasing. This follows from Assumption 1. Therefore, $q_{2}\left(v_{2}\right)$ is also non-decreasing and the optimal solutions of $\mathcal{P}_{1}$ and $\mathcal{P}_{2}$ together fulfill all constraints of (4.3).

In the remainder of this section the features of the optimal solution will be discussed.

Distortions in Both Periods. First, distortions due to the binding deadline incentive constraint arise in both periods. By Theorem $4, \pi_{1}(\bar{U})$ is continuously differentiable. Therefore, $p_{U}>0$ implies that the allocation for $d=1$ is distorted. Hence, the general solution involves a distortion for both deadlines, whenever the relaxed solution is not incentive compatible. As distortions are more costly at the deadline which occurs more frequently, the relative magnitude of the distortions depends on $\rho$. If $d=1$ is relatively unlikely ( $\rho$ small), then the distortion of the fixed price is bigger and the auction is closer to Myerson's solution.

Bunching of Deadlines. For buyer one, the expected utility from participation is independent of the deadline if $v_{1}>\beta$. For both deadlines, the winning probability is one, and the expected payment in the auction is equal to the fixed price in period one. In other words, the optimal allocation does not separate types with different deadlines if their valuations are high. It can be implemented in a mechanism where types with valuations $v_{1}>\beta$ buy immediately, and types with lower valuations wait until their deadline. This finding also holds without Assumption 2. It can be shown that the utility in period one has to satisfy $U_{1}\left(v_{1}, 1\right) \in\left[\max \left\{0, U_{1}(\bar{v}, 2)-\left(\bar{v}-v_{1}\right)\right\}, U_{1}\left(v_{1}, 2\right)\right]$ if the relaxed solution is not incentive compatible. This implies that the incentive constraint for the deadline holds with equality for the highest type. Since the relaxed solution is not incentive compatible, an increase of $U_{1}\left(v_{1}, 1\right)$ for high valuations has a first-order effect on expected revenue. Therefore, the allocation rule for $d=2$ must also be distorted for high valuations which implies $q_{1}\left(v_{1}, 2\right)=1$ for $v_{1}$ sufficiently high. It is likely that bunching of this type also arises in more general models, so that some (high) types are not rewarded for delaying a purchase until their respective deadlines. 
Dominant Strategies and Indirect Implementation. While the optimal allocation conditional on $d=1$ can be implemented by posting fixed prices, there are several ways to implement the optimal auction in period two. For example, it can be implemented by a generalized Vickrey auction. In this auction, which has a payment rule similar to $y^{\text {rlx }}$, the winning bidder pays the valuation for which his (generalized) virtual valuation ties with the (generalized) virtual valuation of the losing bidder. For buyer two, this mechanism is incentive compatible in dominant strategies. ${ }^{37}$ Hence, the optimal mechanism does not rely on the seller's ability to conceal information about period one.

As in the static auction model, there is also an ascending clock auction format that corresponds to this direct mechanism. The auctioneer has a clock that runs continuously from zero to $\bar{v}$. For each bidder $i$, the auctioneer's clock value $c_{a}$ is translated into a bidder-specific clock value $c_{i}$. For bidder one, this is $c_{1}=\left(J_{1}^{p_{U}}\right)^{-1}\left(c_{a}\right)$. For bidder two, this is $c_{2}=J_{2}^{-1}\left(c_{a}\right)$. The auctioneer raises $c_{a}$ continuously and bidders can drop out at any time. If bidder $i$ drops out, the clock stops immediately. Bidder $j \neq i$ wins the object and has to make a payment equal to his bidder-specific clock-value $c_{j}$. Given the informational assumptions made in this paper, this auction is strategically equivalent to the generalized Vickrey auction. It has the advantage that the winning bidder does not have to reveal his true valuation to the auctioneer.

\section{Concluding Remarks}

The results of Section 4 are obtained by solving an optimal auction problem with a type-dependent participation constraints. Similar problems arise in several contexts and (with some limitations) the methods used here may be helpful for such exercises.

First, as mentioned in Section 1.1 and Footnote 7, there are many models with twodimensional private information where the second dimension takes the form of a constraint in the agents' utility functions. So far the literature has focused on the regular case in which incentive constraints in the second dimension can be ignored. The methods used here will be useful for a more general characterization of optimal mechanisms. Second, type dependent participation constraints arise naturally if there is competition. In the context of our model, buyers may find other opportunities to purchase a similar object if they are not awarded in the period of arrival. In this case, patient buyers would face two "outside options"- they can misreport their patience and choose to buy from a different seller. From the perspective of the seller, this leads to stochastic exit or random participation as in Rochet and Stole (2002). In a static context, models of competing auctioneers naturally involve type-dependent participation constraints. Third, heterogeneity in the degree of patience could be incorporated in search models and the present analysis may be useful for the analysis of such applications.

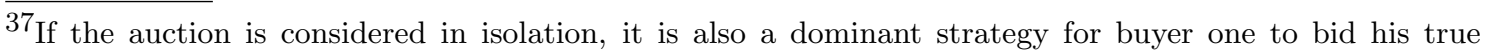
valuation. In the dynamic context, however, it is not a dominant strategy to report the deadline truthfully.
} 
The main limiting factors for a generalization of our methods (e.g., to a model with more than two buyers) result from the combination of the type dependent participation constraint, the monotonicity constraint, and the possibility of discontinuities in the winning probability (at the reserve price). In the proof of Theorem 5 we solve an optimal control problem where the interim winning probability is a state variable with discontinuities, and the controls are its derivative and the position and size of jumps. The type-dependent participation constraint takes the form of a state constraint. We simplify the problem by showing that this constraint has to be imposed only for the highest type (by Assumption 2). ${ }^{38}$ The result then follows from an application of a version of the Pontryagin maximum principle. The Lagrangian relaxation suggest how the optimal mechanism looks like if the participation constraint is imposed for interior types (this may be the case without Assumption 2 or if there are two or more buyers in the first period). ${ }^{39}$ The formal proof amounts to applying the right version of the maximum principle. In other applications, some complication can be avoided if the model is set up such that discontinuities in the winning probability do not arise - for example by ruling out an exclusion region.

Increasing the number of periods introduces an additional complication because buyers from different cohorts with different participation constraints participate simultaneously. In the two-period model, it was possible to use the participation constraint to substitute the winning probability of the buyer who does not face a participation constraint. Applying this technique (recursively) to multiple cohorts is in principle possible, but can become tedious. Therefore it will be more likely to find tractable applications in models with overlapping generations where each cohort lives for at most two periods. Again, one can get an intuition for the optimal solution from a Lagrangian relaxation.

\section{Appendix A. Proofs of Lemmas 2 and 4, and Theorems 3 and 4}

Proof of Lemma 2. By Theorem 4.A.5 in Shaked and Shanthikumar (2007), (weak) secondorder stochastic dominance follows from the martingale property. We strengthen this property and show that for every strictly concave function $\phi$, all $a \leq d<d^{\prime}$, and all $H_{a}^{-i}$ such that $\underline{J}_{a}\left(H_{a}^{-i}\right) \neq \bar{v}$ we have

$$
E\left[\phi\left(\underline{J}_{a}\left(H_{d}^{-i}\right)\right) \mid H_{a}^{-i}\right]>E\left[\phi\left(\underline{J}_{a}\left(H_{d^{\prime}}^{-i}\right)\right) \mid H_{a}^{-i}\right] .
$$

To see that this is true, we use the law of iterated expectations on both sides:

$$
E\left[E\left[\phi\left(\underline{J}_{a}\left(H_{d}^{-i}\right)\right) \mid H_{d}^{-i}\right] \mid H_{a}^{-i}\right]>E\left[E\left[\phi\left(\underline{J}_{a}\left(H_{d^{\prime}}^{-i}\right)\right) \mid H_{d}^{-i}\right] \mid H_{a}^{-i}\right] .
$$

If the distribution of $\left[\phi\left(\underline{J}_{a}\left(H_{d^{\prime}}^{-i}\right)\right) \mid H_{d}^{-i}\right]$ is a Dirac measure, then by the martingale property we have

$$
\left[\phi\left(\underline{J}_{a}\left(H_{d}^{-i}\right)\right) \mid H_{d}^{-i}\right]=\left[\phi\left(\underline{J}_{a}\left(H_{d^{\prime}}^{-i}\right)\right) \mid H_{d}^{-i}\right] .
$$

But if $\underline{J}_{a}\left(H_{a}^{-i}\right) \neq \bar{v}$, there is a positive probability that a history $H_{d}^{-i}$ occurs for which the distribution $\left[\phi\left(\underline{J}_{a}\left(H_{d^{\prime}}^{-i}\right)\right) \mid H_{d}^{-i}\right]$ is not concentrated on a single point. Since $\left[\phi\left(\underline{J}_{a}\left(H_{d}^{-i}\right)\right) \mid H_{d}^{-i}\right]$

\footnotetext{
${ }^{38}$ Pai (2014) uses a similar trick in part of his analysis of competing auctioneers.

${ }^{39}$ See footnote 34 .
} 
is deterministic, weak second-order stochastic dominance together with strict concavity implies that

$$
E\left[\phi\left(\underline{J}_{a}\left(H_{d}^{-i}\right)\right) \mid H_{d}^{-i}\right]>E\left[\phi\left(\underline{J}_{a}\left(H_{d^{\prime}}^{-i}\right)\right) \mid H_{d}^{-i}\right] .
$$

This shows the desired result.

Proof of Theorem 3. Consider a buyer $i$ with type $(a, v, d)$, where $a \leq d<T$ and let $d^{\prime} \in\{d+1, \ldots, T\}$. Fix the state in the arrival period $s_{a}$, and let

$$
\begin{aligned}
G(\underline{J}) & =\operatorname{Prob}\left[\underline{J}_{a}\left(H_{d}^{-i}\right) \leq \underline{J} \mid s_{a}\right], \\
\text { and } \quad G^{\prime}(\underline{J}) & =\operatorname{Prob}\left[\underline{J}_{a}\left(H_{d^{\prime}}^{-i}\right) \leq \underline{J} \mid s_{a}\right] .
\end{aligned}
$$

Lemma 2 implies that the c.d.f.s $G$ and $G^{\prime}$ have the same mean and $G \succ_{\mathrm{SSD}} G^{\prime}$.

(i) Suppose that $J_{a}(v)$ is convex and let $J_{a}(v)>0$. Conditional on $s_{a}$ we have ${ }^{40}$

$$
\begin{aligned}
U_{a}\left(v, d^{\prime} \mid s_{a}\right) & =v G^{\prime}\left(J_{a}(v)\right)-J_{a}^{-1}(0) G^{\prime}(0)-\int_{0}^{J_{a}(v)} J_{a}^{-1}(\underline{J}) d G^{\prime}(\underline{J}), \\
& =\int_{0}^{J_{a}(v)} \frac{d J_{a}^{-1}(\underline{J})}{d \underline{J}} G^{\prime}(\underline{J}) d \underline{J}, \\
& \geq \int_{0}^{J_{a}(v)} \frac{d J_{a}^{-1}(\underline{J})}{d \underline{J}} G(\underline{J}) d \underline{J}=U_{a}\left(v, d \mid s_{a}\right)
\end{aligned}
$$

The last line follows because because $d J_{a}^{-1}(\underline{J}) / d \underline{J}$ is non-negative and non-increasing, and for all non-negative and non-increasing functions $\phi:[0, \bar{v}] \rightarrow \mathbb{R}$, we can show that

$$
\forall x \in[0, \bar{v}]: \quad \int_{0}^{x} \phi(s) G^{\prime}(s) d s \geq \int_{0}^{x} \phi(s) G(s) d s .
$$

For $\phi(s)=\mathbf{1}_{\{s \leq M\}}, M \in[0, \bar{v}],($ A.1) reduces to

$$
\forall x \in[0, M]: \quad \int_{0}^{x} G^{\prime}(s) d s \geq \int_{0}^{x} G(s) d s,
$$

which follows directly from SSD. Since any non-increasing function $\phi:[0, \bar{v}] \rightarrow \mathbb{R}$ can be uniformly approximated by non-increasing step functions (A.1) holds for all non-negative and non-increasing functions.

(ii) Suppose that $v=\bar{v}$ and that $J_{a}(v)$ is strictly concave. Conditional on $s_{a}$ the expected payoffs of $i$ for $d$ and $d^{\prime}$ satisfy

$$
\begin{aligned}
U_{a}\left(\bar{v}, d^{\prime} \mid s_{a}\right) & =\bar{v}-E\left[J_{a}^{-1}\left(\underline{J}_{a}\left(H_{d^{\prime}}^{-i}\right)\right) \mid s_{a}\right] \\
& <\bar{v}-E\left[J_{a}^{-1}\left(\underline{J}_{a}\left(H_{d}^{-i}\right)\right) \mid s_{a}\right]=U_{a}\left(\bar{v}, d \mid s_{a}\right),
\end{aligned}
$$

which violates $\left(\mathrm{ICD}^{\mathrm{d}}\right)$. In the second line we have used strict convexity of $J_{a}^{-1}(\underline{J})$ as a function of $\underline{J}$ and strict second-order stochastic dominance.

Proof of Lemma 4. It is obvious that $\mathcal{U}\left(p_{U}\right)$ is increasing. We have to show that $\mathcal{U}\left(p_{U}\right)$ is upper-hemicontinuous and closed-valued. To see this, we first show that $\bar{M}_{1}^{p_{U}}(t)$ is

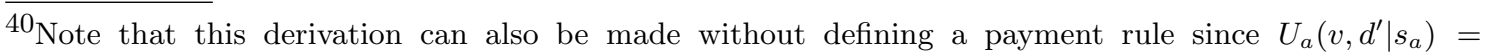
$\int_{0}^{v} q_{d^{\prime}}\left(w, d^{\prime} \mid s_{a}\right) d w=\int_{0}^{J_{a}(v)} G^{\prime}(\underline{J})\left(d J_{a}^{-1}(\underline{J}) / d \underline{J}\right) d \underline{J}$.
} 
continuous in $p_{U}$ for all $t . \quad M_{1}^{p_{U}}(t)=M_{1}(t)+p_{U} v_{1}^{\prime}(t)$ is continuous in $p_{U}$ and $t$ and therefore $\mathcal{M}_{1}^{p_{U}}(t)=\int_{0}^{t} M_{1}^{p_{U}}(s) d s$ is continuous in $p_{U}$ and continuously differentiable in $t$. Note that the convex hull of $\mathcal{M}_{1}^{p_{U}}(t)$ can be written as

$$
\begin{aligned}
\overline{\mathcal{M}}_{1}^{p_{U}}(t)= & \operatorname{conv} \mathcal{M}_{1}^{p_{U}}(t)=\max \left\{\alpha+\beta t \mid(\alpha, \beta) \in \mathcal{C}\left(p_{U}\right)\right\}, \\
\text { where } \mathcal{C}\left(p_{U}\right) & =\left\{\alpha, \beta \geq L \mid \alpha+\beta \theta \leq \mathcal{M}_{1}^{p_{U}}(\theta) \forall \theta \in[0,1]\right\},
\end{aligned}
$$

for some $L>-\infty$ sufficiently small. $\mathcal{C}\left(p_{U}\right)$ is compact-valued and continuous in $p_{U}$. Therefore, by Berge's maximum theorem

$$
\left(\alpha_{p_{U}}^{*}, \beta_{p_{U}}^{*}\right)(t):=\arg \max \left\{\alpha+\beta t \mid(\alpha, \beta) \in \mathcal{C}\left(p_{U}\right)\right\}
$$

is non-empty, compact-valued and upper-hemicontinuous in $p_{U}$. Note that $\beta_{p_{U}}^{*}(t)$ is the subgradient of $\overline{\mathcal{M}}_{1}^{p_{U}}(t)$ at $t$. Since $\mathcal{M}_{1}^{p_{U}}(t)$ is continuously differentiable, $\overline{\mathcal{M}}_{1}^{p_{U}}(t)$ is also continuously differentiable and hence the subgradient is a singleton. Therefore $\bar{M}_{1}^{p_{U}}(t)=$ $\beta_{p_{U}}^{*}(t)$ is continuous in $p_{U}$. Continuity of $\bar{M}_{1}^{p_{U}}(t)$ implies that $\mathcal{U}\left(p_{U}\right)$ is continuous if it is single valued - that is if $\bar{M}_{1}^{p_{U}}(t)=0$ has a unique solution. If $\bar{M}_{1}^{p_{U}}(t)=0$ does not have a unique solution, then a compact interval of values $U(\bar{v})$ can be achieved by varying $\underline{x}_{1}^{0} \in[0,1]$. Hence we have shown that $\mathcal{U}\left(p_{U}\right)$ is closed valued.

Finally, notice that $\max \mathcal{U}\left(p_{U}\right)$ and $\min \mathcal{U}\left(p_{U}\right)$ are obtained by integrating $\bar{q}_{1}$ with $\underline{x}_{1}^{0}=1$ and $\underline{x}_{1}^{0}=0$, respectively. Continuity of $\bar{M}_{1}^{p_{U}}(t)$ as a function of $p_{U}$ therefore implies that $\max \mathcal{U}\left(p_{U}\right)$ is right continuous and $\min \mathcal{U}\left(p_{U}\right)$ is left-continuous everywhere. This establishes upper-hemicontinuity.

\section{Appendix B. Proof of Theorem 5}

It will be convenient to make the changes of variables $t_{1}=F_{1}\left(v_{1}\right)$ and $t_{2}=F_{2}\left(v_{2}\right)$. Defining $v_{1}\left(t_{1}\right):=F_{1}^{-1}\left(t_{1}\right)$ and $v_{2}\left(t_{2}\right):=F_{2}^{-1}\left(t_{2}\right)$, we have

$$
\begin{aligned}
t_{i} & \sim U[0,1] \text { for } i=1,2, \\
v_{1}^{\prime}\left(t_{1}\right) & =\frac{1}{f_{1}\left(v_{1}\left(t_{1}\right)\right)}, \\
\text { and } \quad v_{2}^{\prime}\left(t_{2}\right) & =\frac{1}{f_{2}\left(v_{2}\left(t_{2}\right)\right)},
\end{aligned}
$$

Furthermore, for $i=1,2$ we introduce

$$
\begin{aligned}
q_{i}(t) & =q_{i}\left(v_{i}(t), 2\right), \\
U(t) & =U_{1}\left(v_{1}(t), 2\right), \\
M_{i}(t) & =J_{i}\left(v_{i}(t)\right)=v_{i}(t)-(1-t) v_{i}^{\prime}(t), \\
\text { and } \quad t_{i}^{0} & =M_{i}^{-1}(0) .
\end{aligned}
$$

The objective of the seller becomes

$$
R\left[q_{1}, q_{2}\right]:=\int_{0}^{1} q_{1}(t) M_{1}(t)+q_{2}(t) M_{2}(t) d t .
$$


The following Theorem formulates the feasibility constraint in terms of $q{ }^{41}$

Theorem 6 (Mierendorff, 2011). For $i=1,2$, let $q_{i}:[0,1] \rightarrow[0,1]$ be non-decreasing. $\left(q_{1}, q_{2}\right)$ is the reduced form of a feasible allocation rule if and only if for all $t_{1}, t_{2} \in[0,1]$,

$$
\int_{t_{1}}^{1} q_{1}(t) d t+\int_{t_{2}}^{1} q_{2}(t) d t \leq 1-t_{1} t_{2}
$$

Now we can restate $\mathcal{P}_{2}$ as $\mathcal{P}_{2}^{\prime}$ :

$$
\pi_{2}(\bar{U})=\sup _{\left(q_{1}, q_{2}\right)} R\left[q_{1}, q_{2}\right]
$$

subject to

$$
\begin{array}{ll}
\forall t \in[0,1]: & q_{i}(t) \in[0,1], \\
\forall t>t^{\prime}, & q_{i}(t) \geq q_{i}\left(t^{\prime}\right), \\
\forall t_{1}, t_{2} \in[0,1]: & \int_{t_{1}}^{1} q_{1}(\theta) d \theta+\int_{t_{2}}^{1} q_{2}(\theta) d \theta \leq 1-t_{1} t_{2}, \\
\forall t \in[0,1]: & U(t)=\int_{0}^{t} q_{1}(\theta) v_{1}^{\prime}(\theta) d \theta, \\
\text { and } & U(1) \geq \bar{U} .
\end{array}
$$

Using $q_{i}\left(F_{i}\left(v_{i}\right)\right)=q_{i}\left(v_{i}, 2\right)$, a solution to $\mathcal{P}_{2}$ can be derived easily from a solution to $\mathcal{P}_{2}^{\prime}$.

We can use the (non-standard) constraint (B.5) to eliminate $q_{2}$ from the objective function. For $q_{1}:[0,1] \rightarrow[0,1]$ non-decreasing, define

$$
q_{1}^{-1}(t):= \begin{cases}1 & \text { if } q_{1}(1)<t \\ \inf \left\{\theta \in[0,1] \mid q_{1}(\theta) \geq t\right\} & \text { otherwise. }\end{cases}
$$

Lemma 5. Let $q_{1}:[0,1] \rightarrow[0,1]$ be non-decreasing. Then an optimal solution to

$$
\sup _{q_{2}} \int_{0}^{1} q_{2}(t) M_{2}(t) d t \quad \text { subject to (B.3)-(B.5), }
$$

is given by

$$
q_{2}^{*}(t)= \begin{cases}q_{1}^{-1}(t) & \text { if } t \geq t_{2}^{0} \\ 0 & \text { otherwise }\end{cases}
$$

Proof. (B.4) can be rewritten as

$$
\forall t_{2} \in[0,1]: \quad \int_{t_{2}}^{1} q_{2}(\theta) d \theta \leq \min _{t_{1} \in[0,1]}\left[1-t_{1} t_{2}-\int_{t_{1}}^{1} q_{1}(\theta) d \theta\right] .
$$

On the right-hand side we minimize a convex function. Therefore, the first order condition is sufficient for a minimum and we have $t_{2} \in\left[q_{1}\left(t_{1}^{-}\right), q_{1}\left(t_{1}^{+}\right)\right]$for all $t_{2} \in\left[q_{1}(0), q_{1}(1)\right]$, $t_{1}=0$ if $t_{2}<q_{1}(0)$ and $t_{1}=1$ if $t_{2}>q(1)$. Hence $t_{1}=q_{1}^{-1}\left(t_{2}\right)$ is a minimizer for all $t_{2}$.

\footnotetext{
${ }_{41}$ The characterization is a generalization of Border's (1991) characterization for symmetric allocation rules. Matthews (1984) conjectured the result proved by Border (see also Chen, 1986). For an early application of a special case of the result see Maskin and Riley (1984).
} 
Substituting this into (B.4) yields

$$
\forall t_{2} \in[0,1]: \quad \int_{t_{2}}^{1} q_{2}(\theta) d \theta \leq 1-q_{1}^{-1}\left(t_{2}\right) t_{2}-\int_{q_{1}^{-1}\left(t_{2}\right)}^{1} q_{1}(\theta) d \theta .
$$

$q_{2}^{*}$ fulfills this constraint with equality for all $t_{2} \in[0,1]$.

Now consider an alternative solution $\tilde{q}_{2}$ that differs from $q_{2}^{*}$ on a set of positive measure. If $\tilde{q}_{2}(t)>0$ for some $t<t_{2}^{0}$, then it is not a maximizer. So suppose $\tilde{q}_{2}(t)=0$ for $t<t_{2}^{0}$. By (B.8) we must have $\int_{t}^{1} \tilde{q}_{2}(\theta) d \theta \leq \int_{t}^{1} q_{2}^{*}(\theta) d \theta$ for all $t \in[0,1]$. But this implies that $\int_{0}^{1} \phi(\theta) \tilde{q}_{2}(\theta) d \theta \leq \int_{0}^{1} \phi(\theta) q_{2}^{*}(\theta) d \theta$ for all non-negative and non-decreasing functions $\phi$. (The argument is similar to the one use in the proof of Lemma 2.) Setting $\phi(\theta)=\max \left\{0, M_{2}(\theta)\right\}$ we obtain the desired result.

Using Lemma 5, (B.2) becomes

$$
\int_{0}^{1} q_{1}(t) M_{1}(t) d t+\int_{t_{2}^{0}}^{1} q_{1}^{-1}(t) M_{2}(t) d t
$$

Let us define $\tilde{M}_{2}(t):=\max \left\{0, M_{2}(t)\right\}$. Then the second integral in (B.9) can be written as

$$
\begin{aligned}
\int_{0}^{1} q_{1}^{-1}(t) \tilde{M}_{2}(t) d t & =\int_{0}^{1} \int_{0}^{q_{1}^{-1}(t)} \tilde{M}_{2}(t) d s d t=\int_{0}^{1} \int_{q_{1}(s)}^{1} \tilde{M}_{2}(t) d t d s \\
& =\int_{0}^{1} \tilde{M}_{2}(t) d t-\int_{0}^{1} \int_{0}^{q_{1}(t)} \tilde{M}_{2}(s) d s d t
\end{aligned}
$$

Therefore, we have to maximize

$$
\int_{0}^{1}\left\{q_{1}(t) M_{1}(t)-\int_{0}^{q_{1}(t)} \tilde{M}_{2}(s) d s\right\} d t .
$$

subject to (B.3)-(B.4) and (B.6)-(B.7). This is a control problem with state variables $(U, q)$ and a control variable $u(t) \geq 0$.

The evolution of the state variables is governed by

$$
U^{\prime}(t)=q(t) v^{\prime}(t) \quad \text { and } \quad q^{\prime}(t)=u(t),
$$

where the second equation only holds if $q(t)$ is differentiable at $t$. The non-negativity constraint for $u(t)$ guarantees that $q$ is non-decreasing. We have to allow for (upward) jumps in the state variable $q$. Therefore, we will be looking for solutions $(U, q, u)$ such that $U$ is continuous and piecewise continuously differentiable, $q$ is piecewise continuously differentiable with a finite number of jumps at points $\tau_{1}, \ldots, \tau_{k}$ and $u$ is left-continuous. The remaining constraints of the problem are

$$
q(0)=0, \quad U(0)=0, \quad q(1) \leq 1, \quad \text { and } \quad U(1) \geq \bar{U} .
$$

The Hamiltonian of this problem is

$$
\mathcal{H}\left(U, q, u, p_{U}, p_{q}, t\right)=q M_{1}(t)-\int_{0}^{q} \tilde{M}_{2}(s) d s+p_{U} q v_{1}^{\prime}(t)+p_{q} u
$$


where $p_{U}$ and $p_{q}$ are the adjoint variables of $U$ and $q$, respectively. Since $\partial \mathcal{H} / \partial U=0$, and

$$
\frac{\partial \mathcal{H}}{\partial q}=-\tilde{M}_{2}(q)+M_{1}^{p_{U}}(t)
$$

Assumption 1 implies that $\mathcal{H}$ is concave in $(U, q)$, independently of $u$. Therefore, by Theorems 7 and 8 in Seierstad and Sydsaeter (1987, pp. 196-199) the following conditions are necessary and sufficient for optimality

(i) For all $t$ such that $q$ is continuous, $p_{q}(t) \leq 0$ and

$$
u(t) \begin{cases}=0, & \text { if } p_{q}(t)<0 \\ \in \mathbb{R}_{+}, & \text {if } p_{q}(t)=0\end{cases}
$$

(ii) For all $t$ such that $q$ and $u$ are continuous

$$
\begin{aligned}
p_{U}^{\prime}(t) & =0, \\
p_{q}^{\prime}(t) & =-M_{1}^{p_{U}}(t)+\tilde{M}_{2}(q(t)) .
\end{aligned}
$$

(iii) $p_{q}(0)$ and $p_{U}(0)$ are free and

$$
\begin{aligned}
& p_{U}(1) \geq 0 \quad(\text { with equality if } U(1)>\bar{U}), \\
& p_{q}(1) \leq 0 \quad(\text { with equality if } q(1)<1) .
\end{aligned}
$$

(iv) At all jump-points $\tau_{j}, j=1, \ldots k$, of $q, p_{q}$ and $p_{U}$ are continuous and

$$
p_{q}\left(\tau_{j}\right) \geq 0 .
$$

We want to show optimality of the solution $q^{*}(t)$ that is obtained by applying the transformation in (B.1) to the reduced form of the solution (4.9)-(4.11). $q^{*}$ has at most two jump-points

$$
\begin{aligned}
\tau_{1} & :=\min \left\{t \geq 0 \mid \bar{M}_{1}^{p_{U}^{*}}(t) \geq 0\right\}, \\
\text { and } \quad \tau_{2} & :=\inf \left\{t \geq 0 \mid \bar{M}_{1}^{p_{U}^{*}}(t)>0\right\}
\end{aligned}
$$

where $\bar{M}_{1}^{p_{U}}(t)$ is defined in footnote 36 . If $\bar{M}_{1}^{p_{U}^{*}}(t)>0$ for all $t$, we have $\tau_{1}=\tau_{2}=0$, and if $\bar{M}_{1}^{p_{U}^{*}}(t)=0$ has a unique solution $t>0$ we have $\tau_{1}=\tau_{2}>0$. Furthermore, we set

$$
b:=\min \left\{t \geq 0 \mid \bar{M}_{1}^{p_{U}}(t) \geq \bar{v}\right\}
$$

With these definitions we have that

$$
q^{*}(t)= \begin{cases}0, & \text { if } t<\tau_{1}, \\ \underline{x}_{1}^{0} t_{2}^{0} & \text { if } t \in\left[\tau_{1}, \tau_{2}\right), \\ \tilde{M}_{2}^{-1}\left(\bar{M}_{1}^{p_{U}^{*}}(t)\right) & \text { if } t \in\left[\tau_{2}, b\right) \\ 1 & \text { if } t \geq b\end{cases}
$$


The adjoint variable for $U$ is given by $p_{U}(t)=p_{\bar{U}}^{*}$ for all $t$ and the adjoint variable for $q$ is defined as follows. First we set

$$
p_{q}^{*}(0)=\int_{0}^{\tau_{1}} M_{1}^{p_{U}^{*}}(s) d s \leq 0
$$

which is strictly negative if $\tau_{1}>0$. (At $\tau_{1}$, we have $\int_{0}^{\tau_{1}} M_{1}^{p_{\bar{U}}^{*}}(s) d s=\int_{0}^{\tau_{1}} \bar{M}_{1}^{p_{\bar{U}}^{*}}(s) d s \leq 0$.) Inserting $q^{*}(t)$ into (B.12), and using that $\tilde{M}_{2}\left(q^{*}(t)\right)=0$ if $t<\tau_{1}$, we have that for all $t<\tau_{1}$

$$
p_{q}^{*}(t)=\int_{t}^{\tau_{1}} M_{1}^{p_{U}^{*}}(s) d s<0,
$$

and $p_{q}^{*}\left(\tau_{1}\right)=0$. Therefore, for $t<\tau_{1}, u^{*}(t)=q^{* \prime}(t)=0$ is consistent with (B.11). Next, we note that $p_{q}^{* \prime}(t)=\tilde{M}_{2}\left(q^{*}(t)\right)-M_{1}^{p_{\bar{U}}^{*}}(t)=\bar{M}_{1}^{p_{\bar{U}}^{*}}(t)-M_{1}^{p_{\bar{U}}^{*}}(t)$ for $t \in\left[\tau_{1}, b\right)$ and hence ${ }^{42}$

$$
\begin{aligned}
p_{q}^{*}(t) & =\int_{0}^{t} \bar{M}_{1}^{p_{U}^{*}}(s)-M_{1}^{p_{U}^{*}}(s) d s \\
& =\overline{\mathcal{M}}_{1}^{p_{U}^{*}}(t)-\mathcal{M}_{1}^{p_{U}^{*}}(t) d s \leq 0, \quad \text { for } t \in\left[\tau_{1}, b\right) .
\end{aligned}
$$

If $q^{*}$ is increasing at $t$, we have $\overline{\mathcal{M}}_{1}^{p_{U}^{*}}(t)=\operatorname{conv} \int_{0}^{t} M_{1}^{p_{U}^{*}}(s) d s=\int_{0}^{t} M_{1}^{p_{U}^{*}}(s) d s=\mathcal{M}_{1}^{p_{U}^{*}}(t) d s$, so that $p_{q}^{*}(t)=0$. Hence, for $t \in\left[\tau_{1}, b\right), u^{*}(t)=q^{* \prime}(t)$ is consistent with (B.11) and (B.13). Finally, we have $p_{q}^{*}(b)=0$ and therefore

$$
p_{q}^{*}(t)=\int_{b}^{t} \tilde{M}_{2}(1)-M_{1}^{p_{U}^{*}}(s) d s \leq \int_{b}^{t} \bar{M}_{1}^{p_{U}^{*}}(s)-M_{1}^{p_{U}^{*}}(s) d s=\overline{\mathcal{M}}_{1}^{p_{U}^{*}}(t)-\mathcal{M}_{1}^{p_{U}^{*}}(t) d s \leq 0
$$

for $t \geq b$ so that for $t \geq b u^{*}(t)=q^{*}(t)=0$ is also consistent with (B.11).

To summarize, we have shown that for $\left(U^{*}, q^{*}, u^{*}\right)$, there exist adjoint variables such that the necessary and sufficient conditions are satisfied. This proves part (i) of the Theorem. Part (ii) is obvious. The proofs of parts (iii) and (iv) can be found in Appendix C.1 in the Supplemental Material.

\section{REFERENCES}

Armstrong, M. (1996): "Multiproduct Nonlinear Pricing," Econometrica, 64, 51-75. Athey, S. And I. Segal (2013): "An Efficient Dynamic Mechanism," Econometrica, 81, $2463-2485$.

Battaglini, M. (2005): "Long-Term Contracting with Markovian Consumers," American Economic Review, 95, 637-658.

Beaudry, P., C. Blackorby, and D. Szalay (2009): "Taxes and Employment Subsidies in Optimal Redistribution Programs," American Economic Review, 99, 216-242.

Bergemann, D. And M. Said (2011): "Dynamic Auctions," in Wiley Encyclopedia of Operations Research and Management Science, ed. by J. J. Cochran, Wiley.

Bergemann, D. And J. VÄLimäki (2010): "The Dynamic Pivot Mechanism," Econometrica, 78, 711-789.

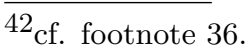


Blackorby, C. And D. Szalay (2008): "Regulating a Monopolist with unknown costs and unknown quality capacity," Warwick Economic Research Papers No. 858.

Board, S. AND A. SkrzypaCz (2015): "Revenue Management with Forward-Looking Buyers," Journal of Political Economy, forthcoming.

Border, K. C. (1991): "Implementation of Reduced Form Auctions: A Geometric Approach," Econometrica, 59, 1175-1187.

Bull, J. And J. WAtson (2007): "Hard Evidence and Mechanism Design," Games and Economic Behavior, 58, 75-93.

Che, Y.-K. And I. Gale (2000): "The optimal Mechanism for Selling to a BudgetConstrained Buyer," Journal of Economic Theory, 92, 198-233.

Chen, Y.-M. (1986): "An extension to the implementability of reduced form auctions," Econometrica, 54, 1249-1251.

Courty, P. And H. Li (2000): "Sequential Screening," Review of Economic Studies, 67, 697-717.

Das Varma, G. and N. Vettas (2001): "Optimal Dynamic Pricing with Inventories," Economics Letters, 72, 335-340.

Deb, R. (2011): "Intertemporal Price Discrimiation with Stochastic Values," Yale University, unpublished working paper.

Dizdar, D., A. Gershkov, and B. Moldovanu (2011): "Revenue Maximization in the Dynamic Knapsack Problem," Theoretical Economics, 6, 157-184.

Elmaghraby, W. and P. Keskinocak (2003): "Dynamic Pricing in the Presence of Inventory Considerations: Research Overview, Current Practices, and Future Directions," Management Science, 49, 1287-1309.

Eso, P. And B. Szentes (2007): "Optimal Information Disclosure in Auctions and the Handicap Auction," Review of Economic Studies, 74, 705-731.

Ewerhart, C. (2013): "Regular Type Distributions in Mechanism Design and $\rho$ concavity," Economic Theory, 53, 591-603.

Gershkov, A. And B. Moldovanu (2009a): "Dynamic Revenue Maximization with Heterogeneous Objects: A Mechanism Design Approach," American Economic Journal: Microeconomics, 1, 168-198.

(2009b): "Learning about the Future and Dynamic Efficiency," American Economic Review, 99, 1576-1588.

(2010): "Incentive Compatible Optimal Stopping," University of Bonn, unpublished working paper.

— (2012a): "Dynamic allocation and pricing: A mechanism design approach," International Journal of Industrial Organization, 30, 283-286.

_ (2012b): "Optimal Search, Learning, and Implementation," Journal of Economic Theory, 147, 881-909.

Green, J. R. And J.-J. Laffont (1986): "Partially Verifiable Information and Mechanism Design," The Review of Economic Studies, 53, 447-456. 
Iyengar, G. And A. Kumar (2008): "Optimal Procurement Auctions for Divisible Goods with Capacitated Suppliers," Review of Economic Design, 12, 129-154.

Jehiel, P., B. Moldovanu, and E. Stacchetti (1999): "Multidimensional Mechanism Design for Auctions with Externalities," Journal of Economic Theory, 85, 258-294.

Jullien, B. (2000): "Participation Constraints in Adverse Selection Models," Jorunal of Economic Theory, 91, 1-47.

Malakhov, A. And R. Vohra (2005): "Optimal Auctions for Asymmetrically Budget Constrainted Bidders," Nothwestern University, unpublished working paper.

Manelli, A. M. and D. R. Vincent (2007): "Multidimensional mechanism design: Revenue maximization and the multiple-good monopoly," Journal of Economic Theory, 137, 153-185.

Maskin, E. AND J. Riley (1984): "Optimal Auctions with Risk Averse Buyers," Econometrica, 52, 1473-1518.

Matthews, S. A. (1984): "On the Implementability of Reduced Form Auctions," Econometrica, 52, 1519-1522.

McAfee, R. P. And V. Te Velde (2007): "Dynamic Pricing in the Airline Industry," in Handbook on Economics and Information Systems, ed. by T. Hendershott, Elsevier, vol. 1 of Handbooks in Information Systems.

Mierendorff, K. (2011): "Asymmetric Reduced Form Auctions," Economics Letters, $110,41-44$.

_ (2013): "The Dynamic Vickrey Auction," Games and Economic Behavior, 82, 192-204.

Möller, M. And M. Watanabe (2010): "Advance Purchase Discounts versus Clearance Sales," The Economic Journal, 120, 1125-1148.

Myerson, R. B. (1981): "Optimal Auction Design," Mathematics of Operations Reseach, $6,58-63$.

- (1998): "Population Uncertainty and Poisson Games," International Journal of Game Theory, 27, 375-392.

Nocke, V. And M. Peitz (2007): "A Theory of Clearance Sales," The Economic Journal, 117, 964-990.

Pai, M. And R. Vohra (2013): "Optimal Dynamic Auctions and Simple Index Rules," Mathematics of Operations Reseach, 38, 682-697.

(2014): "Optimal Auctions with Financially Constrained Bidders," Journal of Economic Theory, 150, 383-425.

PAI, M. M. (2014): "Competing Auctioneers," University of Pennsylvania, unpublished working paper.

Parkes, D. C. And S. Singh (2003): "An MDP-Based Approach to Online Mechanism Design," in Proc. 17th Annual Conf. on Neural Information Processing Systems (NIPS'03).

Pavan, A., I. Segal, And J. Toikka (2014): "Dynamic Mechanism Design: A Myersonian Approach," Econometrica, 82, 601-653. 
Peskir, G. And A. Shiryaev (2006): Opitmal Stopping and Free-Boundary Problems, Birkhäuser Verlag.

ReID, W. T. (1968): "A Simple Optimal Control Problem Involving Approximation by Monotone Functions," Journal of Optimization Theory and Applications, 2, 365-377.

Riley, J. And R. Zeckhauser (1983): "Optimal Selling Strategies: When to Haggle, When to Hold Firm," The Quarterly Journal of Economics, 98, 267-289.

Rochet, J.-C. And P. Choné (1998): "Ironing, Sweeping and Multidimensional Screening," Econometrica, 66, 783-826.

Rochet, J.-C. And L. A. Stole (2002): "Non-linear Pricing with Random Participation," Review of Economic Studies, 69, 277-311.

Seierstad, A. And K. Sydsaeter (1987): Optimal Control Theory with Economic Applications, Elsevier.

Shaked, M. and J. G. Shanthikumar (2007): Stochastic Orders, Springer.

Su, X. (2007): "Intertemporal Pricing with Strategic Customer Behaviour," Management Science, 53, 726-741.

Szalay, D. (2009): "Regulating a Multi-Attribute/Multi-Type Monopolist," University of Bonn, unpublished working paper.

Vulcano, G., G. van Ryzin, and C. Maglaras (2002): "Optimal Dynamic Auctions for Revenue Management," Management Science, 48, 1388-1407. 


\section{Appendix C. Supplemental Material (Online Appendix)}

\section{C.1. Omitted Proofs.}

Proof of Lemma 1. In a first step, we define $\hat{y}$ such that transaction with a buyer only take place at the arrival time and do not depend on whether the object is available at the arrival time. Formally, $\hat{y}_{i}\left(s_{t}\right)=0$ if $t \neq a_{i}$ and $\hat{y}_{i}\left(s_{a_{i}}\right)=q_{a_{i}}\left(v_{i}, d_{i}\right)$. It is straightforward to see that $(x, \hat{y})$ is incentive compatible and individually rational and yields the same revenue as $(x, y)$. The modification allows us to modify the allocation rule without changing the payments that a buyer has to make.

$\hat{x}$ is then derived from $x$ as follows. Whenever a buyer is allocated a unit before his deadline is reached, this allocation is postponed to the deadline. Whenever a buyer is allocated unit after his deadline has elapsed, the unit is withheld under the new allocation rule. In all other cases, the new allocation rule is the same as the old one.

This implies that buyers who report their deadline truthfully enjoy the same expected payoff in both mechanisms:

$$
\forall a \in\{1, \ldots T\}, d \in\{a, \ldots T\}, \forall v \in[0, \bar{v}]: \quad \sum_{\tau=a}^{d} \hat{q}_{a}(v, d)=\sum_{\tau=a}^{d} q_{a}(v, d) .
$$

On the other hand, for $d^{\prime} \neq d$, we have

$$
\sum_{\tau=a}^{d} \hat{q}_{a}\left(v, d^{\prime}\right) \leq \sum_{\tau=a}^{d} q_{a}\left(v, d^{\prime}\right)
$$

Hence,

$$
\hat{U}_{a}(v, d)=U_{a}(v, d) \geq U_{a}\left(v, d, v^{\prime}, d^{\prime}\right) \geq \hat{U}_{a}\left(v, d, v^{\prime}, d^{\prime}\right) .
$$

Proof sketch of Theorem 1. (M) and (PE) is the standard characterization of one-dimensional incentive compatibility for the valuation Myerson (1981). (ICD $\left.{ }^{\mathrm{d}}\right)$ rules out under-reports of the deadline. Together with $(\mathrm{M})$ and $(\mathrm{PE})$, this also rules out simultaneous misreports of an earlier deadline $d^{\prime}<d$ and a valuation $v^{\prime} \neq v$. Since the mechanism never allocates after the reported deadline, the constraint takes this simple form because the utility of under-reporting the deadline is independent of the true deadline (cf. (2.1)):

$$
d^{\prime} \leq d \quad \Rightarrow \quad U_{a}\left(v, d, v^{\prime}, d^{\prime}\right)=U_{a}\left(v, d^{\prime}, v^{\prime}, d^{\prime}\right)
$$

Incentive compatibility for the valuation implies that $U_{a}\left(v, d^{\prime}, v^{\prime}, d^{\prime}\right)$, and therefore also $U_{a}\left(v, d, v^{\prime}, d^{\prime}\right)$, is maximized by $v^{\prime}=v$. For $v^{\prime}=v,\left(\mathrm{ICD}^{\mathrm{d}}\right)$ rules out a downward deviation in the deadline. Therefore, simultaneous deviations in the deadline and the valuation are also ruled out. Necessity of $\left(\mathrm{ICD}^{\mathrm{d}}\right)$ is obvious.

For mechanisms that allocate only at the deadline, reporting $d^{\prime}>d$ can only be profitable if the mechanism pays a subsidy, i.e., if $p_{a}\left(v, d^{\prime}\right)<0$. (PE) implies that subsidies are non-increasing in the valuation. Therefore, the highest subsidy (if any) is paid for $\left(0, d^{\prime}\right)$. By $(\mathrm{PE}), v=0$ is also the valuation for which over-reporting the deadline is most 
tempting. Hence, to rule out upward deviations, it suffices that $U_{a}(0, d)=-p_{a}(0, d) \geq$ $-p_{a}\left(0, d^{\prime}\right)=U_{a}\left(0, d, 0, d^{\prime}\right)=U_{a}\left(0, d^{\prime}\right)$. Together with $\left(\mathrm{ICD}^{\mathrm{d}}\right)$ for $v=0$, this is equivalent to $\left(\mathrm{ICD}^{\mathrm{u}}\right) \cdot{ }^{43}$

Proof of Theorem 5 (iii) and (iv). $\pi_{2}$ can be written as

$$
\pi_{2}(\bar{U})=\int_{0}^{1}\left[q_{p_{\bar{U}}}^{*}(t) M_{1}(t)+\int_{q_{p_{\bar{U}}^{*}}^{*}(t)}^{1} \tilde{M}_{2}(q) d q\right] d t .
$$

We first show that $\pi_{2}(\bar{U})$ is Lipschitz continuous which will be used later. Our assumption on the type distributions guarantee that $M_{1}(t)-\tilde{M}_{2}(q)$ is uniformly bounded for $(t, q) \in$ $[0,1]^{2}$. Therefore, for $\bar{U}^{\prime}>\bar{U}$ we have

$$
\begin{aligned}
& \left|\pi_{2}\left(\bar{U}^{\prime}\right)-\pi_{2}(\bar{U})\right|=\left|\int_{0}^{1} \int_{q_{\bar{U}^{*}}^{*}(t)}^{q_{\bar{U}^{\prime}}^{*}(t)} M_{1}(t)-\tilde{M}_{2}(q) d q d t\right|, \\
& \leq \int_{0}^{1} \int_{q_{\bar{U}}^{*}(t)}^{q_{U^{\prime}}^{*}(t)} \underbrace{\left|M_{1}(t)-\tilde{M}_{2}(q)\right|}_{|.| \leq M<\infty} d q d t, \\
& \leq M \int_{0}^{1} q_{\bar{U}^{\prime}}^{*}(t)-q_{\bar{U}}^{*}(t) d t, \\
& =M\left(\bar{U}^{\prime}-\bar{U}\right) \text {. }
\end{aligned}
$$

Next we show that $\pi_{2}^{\prime}(\bar{U})=-p_{\bar{U}}$. Suppose first that $\bar{U}$ is on the interior of $\mathcal{U}\left(p_{\bar{U}}\right)$ - that is, for $p_{\bar{U}}, \mathcal{U}$ is not single-valued. In this case, $p_{\bar{U}}$ is constant at $\bar{U}$ and the allocation rule only changes on the interval $\left[\tau_{1}, \tau_{2}\right]$ because $\underline{x}_{1}^{0}$ changes with $\bar{U}$. Since

$$
\bar{U}=\underline{x}_{1}^{0} t_{2}^{0}\left(\tau_{2}-\tau_{1}\right)+\int_{\tau_{2}}^{1} q_{p_{\bar{U}}}^{*}(t) d t
$$

we have

Therefore we have

$$
\frac{d x_{1}^{0}}{d \bar{U}}=\frac{1}{t_{2}^{0}\left(\tau_{2}-\tau_{1}\right)}
$$

$$
\begin{aligned}
\frac{d \pi_{2}(\bar{U})}{d \bar{U}} & =\int_{\tau_{1}}^{\tau_{2}} \frac{d \underline{x}_{1}^{0}}{d \bar{U}} t_{2}^{0} M_{1}(t) d t \\
& =-p_{\bar{U}}+\frac{1}{\tau_{2}-\tau_{1}} \int_{\tau_{1}}^{\tau_{2}} M_{1}^{p_{\bar{U}}}(t) d t \\
& =-p_{\bar{U}}+\frac{1}{\tau_{2}-\tau_{1}} \int_{\tau_{1}}^{\tau_{2}} \bar{M}_{1}^{p_{\bar{U}}}(t) d t \\
& =-p_{\bar{U}} .
\end{aligned}
$$

\footnotetext{
${ }^{43}$ If $v \in[\underline{v}, \bar{v}]$ with $\underline{v}>0$, then the upward incentive compatibility constraint for the deadline would be $U_{a}(\underline{v}, d) \geq-p_{a}(\underline{v}, d+1)=U_{a}(\underline{v}, d+1)-q_{a}(\underline{v}, d+1) \underline{v}$. In this case, a subsidy could be used to separate buyers with different deadlines. One can show, however, that this instrument would not be used in the optimal mechanism, unless the allocation rule is sufficiently distorted. The reason is that the cost of a subsidy is of first order whereas the cost of distorting the allocation rule is of second order.
} 
for all $\bar{U}$ that are in the interior of $\mathcal{U}\left(p_{\bar{U}}\right)$. Moreover, if $\mathcal{U}\left(p_{\bar{U}}\right)$ has non-empty interior and $\bar{U}$ is the lower (upper) bound of $\mathcal{U}\left(p_{\bar{U}}\right)$, then the right (left) derivative of $\pi_{2}$ at $\bar{U}$ is equal to $-p_{U}$.

Next suppose that $\mathcal{U}\left(p_{\bar{U}}\right)$ is single valued for $\bar{U}$. For $h>0$, we have

$$
\begin{aligned}
\frac{1}{h}\left(\pi_{2}(\bar{U}+h)-\pi_{2}(\bar{U})\right)= & \frac{1}{h} \int_{0}^{1} \int_{q_{p_{\bar{U}}}^{*}(t)}^{q_{p_{\bar{U}}+h}^{*}(t)} M_{1}(t)-\tilde{M}_{2}(q) d q d t \\
= & \frac{1}{h} \int_{\tau_{1}(\bar{U}+h)}^{b(\bar{U})} \int_{q_{p_{\bar{U}}}^{*}(t)}^{q_{\bar{P}}^{*}+h}{ }^{(t)} M_{1}(t)-\tilde{M}_{2}(q) d q d t, \\
= & -p_{\bar{U}} \frac{1}{h} \underbrace{\int_{\tau_{1}(\bar{U}+h)}^{b(\bar{U})} \int_{q_{p_{\bar{U}}}^{*}(t)}^{q_{p_{\bar{U}}+h}^{*}}(t) v_{1}^{\prime}(t) d q d t}_{=h}+ \\
& +\int_{\tau_{1}(\bar{U}+h)}^{b(\bar{U})} \frac{1}{h} \int_{q_{p_{\bar{U}}}^{*}(t)}^{q_{p_{\bar{U}}+h}^{*}(t)} M_{1}^{p_{U}}(t)-\tilde{M}_{2}(q) d q d t .
\end{aligned}
$$

A similar expression can be derived for $h<0 . \tau_{1}$ and $b$ are are continuous in $\bar{U}$ for almost every $\bar{U}$ (for all $\bar{U}$ if $M_{1}^{p_{U}}$ is strictly increasing). Hence, by the Lebesgue differentiation theorem and dominated convergence, for almost every $\bar{U}$ (every $\bar{U}$ if $M_{1}^{p_{U}}$ is strictly increasing),

$$
\begin{aligned}
\pi_{2}^{\prime}(\bar{U})=\lim _{h \rightarrow 0} \frac{1}{h}\left(\pi_{2}(\bar{U}+h)-\pi_{2}(\bar{U})\right) & =-p_{\bar{U}}+\int_{\tau_{1}}^{b} M_{1}^{p_{\bar{U}}}(t)-\tilde{M}_{2}\left(q_{p_{\bar{U}}}^{*}(t)\right) d t \\
& =-p_{\bar{U}}+\int_{\tau_{1}}^{b} \bar{M}_{1}^{p_{\bar{U}}}(t)-\tilde{M}_{2}\left(q_{p_{\bar{U}}}^{*}(t)\right) d t \\
& =-p_{\bar{U}} .
\end{aligned}
$$

Since $\pi_{2}(\bar{U})$ is Lipschitz continuous it is absolutely continuous and $\pi_{2}(\bar{U})=\pi_{2}(0)-$ $\int_{0}^{\bar{U}} p_{U}(s) d s$. Therefore, as $p_{U}(\bar{U})$ is non-decreasing, $\pi_{2}$ is weakly concave. If $\left\{t \mid \bar{M}_{1}^{p_{U}}(t)\right.$ $=0\}$ is a singleton $p_{U}(\bar{U})$ is strictly increasing an hence $\pi_{2}$ strictly concave.

C.2. Extension of Lemma 2 to the Case of Many Objects. In order to show that Lemma 2 holds for $T=2$ and $K \geq 2$, we need some additional notation. A state is now given by $s_{t}=\left(H_{t}, K_{t}\right)$ where $K_{t}$ is the remaining number of available objects.

For a given state $s_{t}$, define $c_{(1)}^{t} \geq \ldots \geq c_{(K)}^{t}$ as the $K$ highest virtual valuations among the buyers $i \in I_{\leq t}$ with deadlines $d_{i}=t$. If $K_{2}$ units are available in period two, we have

$$
V_{2}\left(H_{2}, K_{2}\right)=\sum_{k=1}^{K_{2}} \max \left\{0, c_{(k)}^{2}\right\} .
$$

The marginal expected revenue in period two from the $k^{\text {th }}$ unit is given by

$$
\begin{aligned}
\Delta\left(s_{1}, k\right) & =E_{s_{2}}\left[V_{2}\left(s_{2}\right) \mid s_{1}, K_{2}=k\right]-E_{s_{2}}\left[V_{2}\left(s_{2}\right) \mid s_{1}, K_{2}=k-1\right] \\
& =E_{s_{2}}\left[\max \left\{0, c_{(k)}^{2}\right\} \mid s_{1}\right] .
\end{aligned}
$$


Obviously, $\Delta\left(s_{1}, k\right)$ is decreasing in $k$. Hence, the optimal number of units to be retained for period two is determined by

$$
\text { and } \begin{aligned}
c_{\left(K-K_{2}^{*}\right)}^{1} & >\Delta\left(s_{1}, K_{2}^{*}+1\right) \quad \text { if } K_{2}^{*}<K, \\
c_{\left(K-K_{2}^{*}+1\right)}^{1} & \leq \Delta\left(s_{1}, K_{2}^{*}\right) \quad \text { if } K_{2}^{*}>0 .
\end{aligned}
$$

If we denote the identities of the buyers with virtual valuations $c_{(1)}^{t}, \ldots, c_{(K)}^{t}$ by $i_{(1)}^{t}, \ldots, i_{(K)}^{t}$, the set of winning buyers in periods $t=1,2$ is given by

$$
\begin{aligned}
& W_{1}^{*}\left(s_{1}\right):=\left\{i_{(1)}^{1}, \ldots, i_{\left(K-K_{2}^{*}\left(s_{1}\right)\right)}^{1}\right\}, \\
& W_{2}^{*}\left(s_{2}\right):=\left\{i_{(1)}^{2}, \ldots, i_{\left(K_{2}^{*}\left(s_{1}\right)\right)}^{2}\right\} \cap\left\{i \in I_{\leq 2} \mid J_{a_{i}}\left(v_{i}\right) \geq 0\right\} .
\end{aligned}
$$

Now we fix some buyer $i \in I_{1}$. His virtual valuation determines whether he is in the set of winning buyers at his deadline $d_{i}$ for a given number of retained objects $K_{2}$, but it may also have an influence on the number of retained objects. The critical virtual valuation is given by

$$
\begin{aligned}
\underline{J}_{1}\left(H_{1}^{-i}\right) & =\inf \left\{J \mid i \in W_{1}^{*}\left(\left(\left(H_{1}^{-i},\left(1, J_{a}^{-1}(J), 1\right)\right), K\right)\right\},\right. \\
& =\inf \left\{J \mid J \geq c_{\left(K-K_{2}^{*}\left(H_{1}^{-i},\left(1, J_{a}^{-1}(J), 1\right)\right)\right)}^{1}\right\},
\end{aligned}
$$

for $d_{i}=1$, and by

$$
\begin{aligned}
\underline{J}_{1}\left(H_{2}^{-i}\right) & =\inf \left\{J \mid i \in W_{2}^{*}\left(\left(H_{2}^{-i},\left(1, J_{a}^{-1}(J), 2\right)\right), K_{2}^{*}\left(H_{1}^{-i},\left(1, J_{1}^{-1}(J), 2\right)\right)\right)\right\}, \\
& =\inf \left\{J \mid \zeta \geq c_{\left(K_{2}^{*}\left(\left(H_{1}^{-i},\left(1, J_{a}^{-1}(J), 1\right)\right)\right)\right.}^{2}\right\},
\end{aligned}
$$

for $d_{1}=2$.

Proof of Lemma 2 for $T=2$ and $K \geq 2$. Let $c_{(k)}^{t,-i}$ denote the $\mathrm{k}^{\text {th }}$ highest virtual valuation among the buyers with deadline $t$ in $I_{\leq t} \backslash\{i\}$. Fix any state $s_{1}=\left(H_{1}, K\right)$ and a buyer $i \in I_{1}$. Suppose that in state $\left(H_{1}^{-i}, K-1\right)$, i.e., if buyer $i$ is not present and one objects less is available, $\bar{K}_{1}$ objects are allocated in period one and $\bar{K}_{2}=K-\bar{K}_{1}$ objects are retained for period two. We distinguish two sub-cases.

Case $A-$ In state $\left(H_{1}^{-i}, K\right), \bar{K}_{1}$ units are allocated in the first period and $\bar{K}_{2}+1$ units are retained for the second period: This implies that

$$
E_{s_{2}}\left[\max \left\{0, c_{\left(\bar{K}_{2}+1\right)}^{2,-i}\right\} \mid H_{1}^{-i}\right] \geq c_{\left(\bar{K}_{1}+1\right)}^{1,-i} .
$$

If in state $\left(\left(H_{1}^{-i},\left(1, v_{i}, d_{i}=1\right)\right), K\right)$, buyer $i$ gets a unit in the first period, then the remaining $K-1$ units are allocated as in state $\left(H_{1}^{-i}, K-1\right)$. This means that $\bar{K}_{1}$ units are allocated to buyers other than $i$ in period one and $\bar{K}_{2}$ units are retained. Hence, $i$ 's virtual valuation must exceed the option value of retaining the $\bar{K}_{2}+1^{\text {st }}$ unit in order to get a unit in the first period. We have

$$
\underline{J}_{1}\left(H_{1}^{-i}\right)=E_{s_{2}}\left[\max \left\{0, c_{\left(\bar{K}_{2}+1\right)}^{2,-i}\right\} \mid H_{1}^{-i}\right]
$$


In state $\left(\left(H_{1}^{-i},\left(1, v_{i}, d_{i}=2\right)\right), K\right)$, the number of units that are allocated in the first period must also be $\bar{K}_{1}$. It is obvious that the arrival of buyer $i$ with $d_{i}=2$ cannot increase the number of units allocated in the first period. On the other hand, suppose that in state $\left(\left(H_{1}^{-i},\left(1, v_{i}, 2\right)\right), K\right)$, only $\bar{K}_{1}-1$ units are allocated in the first period. Then

$$
\begin{aligned}
c_{\left(\bar{K}_{1}\right)}^{1,-i} & \leq E_{s_{2}}\left[\max \left\{0, c_{\left(\bar{K}_{2}+2\right)}^{2}\right\} \mid\left(H_{1}^{-i},\left(1, v_{i}, 2\right)\right)\right], \\
& \leq E_{s_{2}}\left[\max \left\{0, c_{\left(\bar{K}_{2}+2\right)}^{2}\right\} \mid\left(H_{1}^{-i},(1, \bar{v}, 2)\right)\right], \\
& =E_{s_{2}}\left[\max \left\{0, c_{\left(\bar{K}_{2}+1\right)}^{2}\right\} \mid H_{1}^{-i}\right], \\
& <c_{\left(\bar{K}_{1}\right)}^{1,-i},
\end{aligned}
$$

where the last inequality follows from our assumption that in state $\left(H_{1}^{-i}, K-1\right), \bar{K}_{1}$ units are allocated in the first period. This is a contradiction. But if $\bar{K}_{1}$ objects are allocated in the first period, then

$$
\underline{J}_{1}\left(H_{2}^{-i}\right)=\max \left\{0, c_{\left(\bar{K}_{2}+1\right)}^{2,-i}\right\} .
$$

Hence, in case A, $E_{s_{2}}\left[\underline{J}_{1}\left(H_{2}^{-i}\right) \mid H_{1}^{-i}\right]=\underline{J}_{1}\left(H_{1}^{-i}\right)$ and $\left[\underline{J}_{1}\left(H_{1}^{-i}\right) \mid H_{1}^{-i}\right] \succ \operatorname{SSD}\left[\underline{J}_{1}\left(H_{2}^{-i}\right) \mid H_{1}^{-i}\right]$.

Case $B$-In state $\left(H_{1}^{-i}, K\right), \bar{K}_{1}+1$ objects are allocated in the first period and $\bar{K}_{2}$ objects are retained for the second period: In this case,

$$
E_{s_{2}}\left[\max \left\{0, c_{\left(\bar{K}_{2}+1\right)}^{2,-i}\right\} \mid H_{1}^{-i}\right] \leq c_{\left(\bar{K}_{1}+1\right)}^{1,-i} .
$$

Again, if in state $\left(\left(H_{1}^{-i},\left(1, v_{i}, 1\right)\right), K\right)$, buyer $i$ gets an object in the first period, then the remaining $K-1$ objects are allocated as in state $\left(H_{1}^{-i}, K-1\right)$. Hence, in case B we have

$$
\underline{J}_{1}\left(H_{1}^{-i}\right)=c_{\left(\bar{K}_{1}+1\right)}^{1,-i} .
$$

In state $\left(\left(H_{1}^{-i},\left(1, v_{i}, 2\right)\right), K\right)$, it depends on $v_{i}$, how many objects are retained for the second period. Define $z$ by

$$
c_{\left(\bar{K}_{1}+1\right)}^{1,-i}=E_{s_{2}}\left[\max \left\{0, c_{\left(\bar{K}_{2}+1\right)}^{2}\right\} \mid\left(H_{1}^{-i},\left(1, J_{1}^{-1}(z), 2\right)\right)\right] .
$$

$z$ is the lowest virtual value for buyer $i$ such that $\bar{K}_{2}+1$ objects are retained for the second period if $d_{i}=2\left(z\right.$ depends on $H_{1}^{-i}$ !). If $J_{1}\left(v_{i}\right) \geq z$, then $\bar{K}_{2}+1$ objects are retained, otherwise only $\bar{K}_{2}$ objects are retained. Hence, we have

$$
\underline{J}_{1}\left(H_{2}^{-i}\right)= \begin{cases}c_{\left(\bar{K}_{2}+1\right)}^{2,-i}, & \text { if } z<c_{\left(\bar{K}_{2}+1\right)}^{2,-i}, \\ z & \text { if } c_{\left(\bar{K}_{2}+1\right)}^{2,-i} \leq z<c_{\left(\bar{K}_{2}\right)}^{2,-i}, \\ c_{\left(\bar{K}_{2}\right)}^{2,-i} & \text { if } c_{\left(\bar{K}_{2}\right)}^{2,-i} \leq z\end{cases}
$$

Note that for $H_{1}=\left(H_{1}^{-i},\left(1, J_{1}^{-1}(z), 2\right)\right)$ this equals $\max \left\{0, c_{\left(\bar{K}_{2}+1\right)}^{2}\right\}$. So we have

$$
\begin{aligned}
E\left[\underline{J}_{1}\left(H_{2}^{-i}\right) \mid H_{1}^{-i}\right] & =E_{s_{2}}\left[\max \left\{0, c_{\left(\bar{K}_{2}+1\right)}^{2}\right\} \mid\left(H_{1}^{-i},\left(1, J_{1}^{-1}(z), 2\right)\right)\right], \\
& =c_{\left(\bar{K}_{1}+1\right)}^{1,-i}, \\
& =\underline{J}_{1}\left(H_{1}^{-i}\right),
\end{aligned}
$$


and hence $\left[\underline{J}_{1}\left(H_{1}^{-i}\right) \mid H_{1}^{-i}\right] \succ \operatorname{SSD}\left[\underline{J}_{1}\left(H_{2}^{-i}\right) \mid H_{1}^{-i}\right]$.

C.3. Reduced Forms . The probability that a buyer who has arrived in period $a_{i}$, assesses for the event that the number of arrivals in period $a_{i}$ is $N_{a_{i}}$, is given by ${ }^{44}$

$$
\frac{N_{a_{i}} \nu_{a_{i}, N_{a_{i}}}}{\sum_{r=1}^{\infty} r \nu_{a_{i}, r}} .
$$

The interim winning probability for period $t$ of a buyer with type $\left(a_{i}, v_{i}, d_{i}\right)$ is given by:

$$
\begin{aligned}
q_{a_{i}}^{t}\left(v_{i}, d_{i}\right)= & \sum_{\left(N_{1}, \ldots, N_{t}\right) \in \mathbb{N}_{0}^{t}}\left(\frac{N_{a_{i}} \nu_{a_{i}, N_{a_{i}}}}{\sum_{r=1}^{\infty} r \nu_{a_{i}, r}} \prod_{a \in\{1, \ldots, t\} \backslash a_{i}} \nu_{a, N_{a}}\right)\left[\sum_{d_{1}=a_{1}}^{T} \ldots \sum_{d_{i-1}=a_{i-1}}^{T}\right. \\
& \sum_{d_{i+1}=a_{i+1}}^{T} \ldots \sum_{d_{N_{\leq t}}=a_{N_{\leq t}}}^{T}\left(\prod_{j \in I_{\leq t} \backslash i} \rho_{a_{j}, d_{j}}\right) \int_{v_{1} \ldots v_{i-1}} \int_{v_{i+1} \ldots v_{N_{\leq t}}}\left(x_{i}\left(s_{1}\right)+x_{0}\left(s_{1}\right)[\right. \\
& \left.\left.\left.\ldots x_{i}\left(s_{T}\right)\right]\right) \prod_{j \in I_{\leq t} \backslash i} f_{a_{j}}\left(v_{j} \mid d_{j}\right) d v_{j}\right] .
\end{aligned}
$$

The interim expected payment of a buyer with type $\left(a_{i}, v_{i}, d_{i}\right)$ is given by:

$$
\begin{aligned}
p_{a_{i}}\left(v_{i}, d_{i}\right)= & \sum_{\left(N_{1}, \ldots, N_{t}\right) \in \mathbb{N}_{0}^{t}}\left(\frac{N_{a_{i}} \nu_{a_{i}, N_{a_{i}}}}{\sum_{r=1}^{\infty} r \nu_{a_{i}, r}} \prod_{a \in\{1, \ldots, t\} \backslash a_{i}} \nu_{a, N_{a}}\right)\left[\sum_{d_{1}=a_{1}}^{T} \ldots \sum_{d_{i-1}=a_{i-1}}^{T}\right. \\
& \sum_{d_{i+1}=a_{i+1}}^{T} \ldots \sum_{d_{N_{\leq t}}=a_{N_{\leq t}}}^{T}\left(\prod_{j \in I_{\leq t} \backslash i} \rho_{a_{j}, d_{j}}\right) \int_{v_{1} \ldots v_{i-1}} \int_{v_{i+1} \ldots v_{N_{\leq t}}}\left(\sum_{j \in I_{1} \cup\{0\}} x_{j}\left(s_{1}\right) y_{i}\left(s_{1}, k_{2}=j\right)+\right. \\
+ & x_{0}\left(s_{1}\right)\left[\sum_{j \in I_{\leq 2} \cup\{0\}} x_{j}\left(s_{2}\right) y_{i}\left(s_{2}, k_{3}=j\right)+x_{0}\left(s_{3}\right)[\ldots\right. \\
& \left.\left.\left.\left.\sum_{j \in I_{\leq T} \cup\{0\}} x_{j}\left(s_{T}\right) y_{i}\left(s_{T}, k_{T+1}=j\right)\right]\right]\right) \prod_{j \in I_{\leq t} \backslash i} f_{a_{j}}\left(v_{j} \mid d_{j}\right) d v_{j}\right] .
\end{aligned}
$$

C.4. The (lack of a) Relationship between the Martingale Property and Martingale Results from Optimal Stopping. It is well known that the value of the stopped sequence in an optimal stopping problem is a martingale (see for example Theorem 1.2 in Peskir and Shiryaev, 2006). To clarify the relationship between this result and Lemma 2, we provide an example that demonstrates two observations: First, the critical virtual valuation is equal in expectation to the expected virtual value of allocating to the remaining buyers, where the expectation is taken conditional on the history at the arrival of the buyer. Note that the allocation problem of the seller is an optimal stopping problem where the reward process is given be the highest virtual valuation available in a given period. The stopped sequence in this problem is a martingale. Given the first observation one could therefore hope to obtain Lemma 2 from the martingale property of the stopped

\footnotetext{
${ }^{44}$ See Myerson (1998) for a derivation of this expression.
} 
sequence. The second observation in the example, however, shows that the realized critical virtual valuation of a buyer $i$ is different from the stopped sequence derived from the seller's problem of allocation to buyers $j \neq i$. Therefore, it seems that Lemma 2 is not a simple consequence of known results from dynamic programming.

Example 1. Consider a special case of the model with two time period $(T=2)$. Suppose that two buyers arrive in period on $\left(I_{1}=\{1,2\}\right)$, and one buyer arrives in period two $\left(I_{3}=\{3\}\right)$. For simplicity, let us assume that the second buyer in period one is known to be impatient $\left(d_{2}=1\right)$.

Let us first consider the seller's optimal allocation problem when buyer one is not available. This is an optimal stopping problem in finite time. The reward of stopping at $t=1$ is $\max \left\{0, J_{1}\left(v_{2}\right)\right\}$ and the reward of stopping at $t=2$ is $\max \left\{0, J_{2}\left(v_{3}\right)\right\}$. The optimal policy is to stop at $t=1$ if $J_{1}\left(v_{2}\right) \geq E\left[\max \left\{0, J_{2}\left(v_{3}\right)\right\}\right]$, and to stop at $t=2$ otherwise. The value of the stopped process is thus

$$
\begin{aligned}
W & :=\mathbf{1}_{\left\{J_{1}\left(v_{2}\right) \leq \xi(0)\right\}} \max \left\{0, J_{2}\left(v_{3}\right)\right\}+\mathbf{1}_{\left\{J_{1}\left(v_{2}\right)>\xi(0)\right\}} J_{1}\left(v_{2}\right), \\
\text { where } \quad \xi(x) & :=E\left[\max \left\{x, J_{2}\left(v_{3}\right)\right\}\right] .
\end{aligned}
$$

Clearly the process $\left(Z_{1}, Z_{2}\right)$ defined by

$$
Z_{t}=E\left[W \mid H_{t}\right]
$$

is a martingale (this is obvious here but also an implication of a more general result, see e.g., Theorem 1.2 in Peskir and Shiryaev, 2006).

Next, consider the critical virtual valuation of buyer $i=1$. If $d_{1}=1$, it is given by

$$
\underline{J}_{1}\left(H_{1}^{-1}\right)=\max \left\{0, J_{1}\left(v_{2}\right), E\left[\max \left\{0, J_{2}\left(v_{3}\right)\right\}\right]\right\}=\max \left\{J_{1}\left(v_{2}\right), \xi(0)\right\}=Z_{1} .
$$

If $d_{1}=2$, the critical virtual valuation is obtained as follows: With deadline two, buyer one wins if: (a) he has a non-negative virtual valuation, (b) it is not optimal to allocate in period one - that is, $J_{1}\left(v_{2}\right) \leq \xi\left(J_{1}\left(v_{1}\right)\right)$, and (c) buyer three has a lower virtual valuation than buyer one - that is, $J_{2}\left(v_{3}\right) \leq J_{1}\left(v_{1}\right)$. Combining these three conditions, we obtain

$$
\underline{J}_{1}\left(H_{2}^{-1}\right)=\max \left\{0, \xi^{-1}\left(J_{1}\left(v_{2}\right)\right), J_{2}\left(v_{3}\right)\right\} .
$$

The first observation is that $E\left[\underline{J}_{1}\left(H_{2}^{-1}\right) \mid H_{1}\right]=Z_{1}=E\left[Z_{2} \mid H_{1}\right]$ :

$$
\begin{aligned}
E\left[\underline{J}_{1}\left(H_{2}^{-1}\right) \mid H_{1}\right]= & \mathbf{1}_{\left\{J_{1}\left(v_{2}\right) \leq \xi(0)\right\}} E\left[\max \left\{0, J_{2}\left(v_{3}\right)\right\} \mid H_{1}\right] \\
& +\mathbf{1}_{\left\{J_{1}\left(v_{2}\right)>\xi(0)\right\}} E\left[\max \left\{\xi^{-1}\left(J_{1}\left(v_{2}\right)\right), J_{2}\left(v_{3}\right)\right\} \mid H_{1}\right] \\
= & \mathbf{1}_{\left\{J_{1}\left(v_{2}\right) \leq \xi(0)\right\}} \xi(0)+\mathbf{1}_{\left\{J_{1}\left(v_{2}\right)>\xi(0)\right\}} \xi\left(\xi^{-1}\left(J_{1}\left(v_{2}\right)\right)\right) \\
= & \max \left\{J_{1}\left(v_{2}\right), \xi(0)\right\}=Z_{1} .
\end{aligned}
$$

The second observation is that

$$
J_{1}\left(v_{2}\right) \leq \xi(0) \Rightarrow\left\{\begin{array}{ll}
\underline{J}_{1}\left(H_{2}^{-1}\right) & =\max \left\{0, J_{2}\left(v_{3}\right)\right\} \\
Z_{2} & =\max \left\{0, J_{2}\left(v_{3}\right)\right\}
\end{array},\right.
$$




$$
J_{1}\left(v_{2}\right)>\xi(0) \Rightarrow \begin{cases}\underline{J}_{1}\left(H_{2}^{-1}\right) & =\max \left\{\xi^{-1}\left(J_{1}\left(v_{2}\right)\right), J_{2}\left(v_{3}\right)\right\} \\ Z_{2} & =J_{1}\left(v_{2}\right)\end{cases}
$$

Clearly, $\underline{J}_{1}\left(H_{2}^{-1}\right) \neq Z_{2}$. This shows that the martingale property of the generalized virtual valuation cannot be deduced from the martingale property of the stopped sequence in the stopping problem that arises when allocating optimally to buyer $j \neq 1$. To be sure, there may be other ways to obtain Lemma 2 from dynamic programming results, but given the first observation and $\underline{J}_{1}\left(H_{1}^{-1}\right)=Z_{1}$, the approach taken here, seems to be the best candidate. The author has not found other (more fruitful) ways to employ results from dynamic programming.

C.5. Monotonicity and Incentive Compatibility with Private Deadlines. To conclude the analysis of the relaxed solution, we discuss another potential approach to finding sufficient conditions for incentive compatibility of the relaxes solution.

In the standard one-dimensional mechanism design framework, monotonicity of the interim winning probability w.r.t. the valuation is necessary and sufficient for incentive compatibility. It is easy to see, however, that in the dynamic model, monotonicity of the winning probability w.r.t. the deadline is not necessary for incentive compatibility. The following example illustrates this:

Example (continued). Suppose again that Let $T=2$, and $\nu_{1}(1)=\nu_{2}(1)=1$. Moreover, assume that $v_{1}, v_{2} \sim U[0,1]$. This implies that $J_{1}(v)=J_{2}(v)=2 v-1$. If $d_{1}=1$, the option value of storing the object is equal to $E\left[\max \left\{0, J_{2}\left(v_{2}\right)\right\}\right]=\int_{\frac{1}{2}}^{1} 2 v-1 d v=1 / 4$. Therefore, we have $q_{1}^{\mathrm{rlx}}\left(v_{1}, d_{1}=1\right)=1$ for $v_{1} \geq 5 / 8$, and $q_{1}^{\mathrm{rlx}}\left(v_{1}, d_{1}=1\right)=0$ otherwise. If $d_{1}=2$, however, buyer one wins if $2 v_{1}-1 \geq \max \left\{0,2 v_{2}-1\right\}$ so that $q_{1}^{\mathrm{rlx}}\left(v_{1}, d_{1}=2\right)=0$ if $v_{1}<1 / 2$ and $q_{1}^{\mathrm{rlx}}\left(v_{1}, d_{1}=2\right)=v_{1}$ if $v_{1} \geq 1 / 2$. Therefore, $q_{1}^{\mathrm{rlx}}\left(v_{1}, d_{1}\right)$ is decreasing in $d_{1}$ for $v_{1}>5 / 8$ which violates monotonicity. Nevertheless, the relaxed solution is implementable because the virtual valuations of both buyers are linear.

Despite this example, finding conditions that guarantee monotonicity is a natural first approach if we want to find a sufficient condition for incentive compatibility of the relaxed solution. Pai and Vohra (2013) point out that a sufficient condition for $\left(\mathrm{ICD}^{\mathrm{d}}\right)$ is that $q_{a}(v, d)$ is non-decreasing as a function of $d$ for all $a$ and $v$. Together with the envelope formula and $\left(\mathrm{ICD}^{\mathrm{u}}\right)$, this immediately implies that $\left(\mathrm{ICD}^{\mathrm{d}}\right)$ is fulfilled. Monotonicity of $q_{a}(v, d)$, however, is a very strong requirement. Sufficient conditions on the primitives of the model that guarantee monotonicity are not available and indeed, the following example shows that monotonicity in $d$ is unlikely to hold for the relaxed solution.

Example (continued). Let us return to the case of general distributions for the valuations of buyers one and two. Consider the interim winning probability of the buyer who arrives in the first period. If his deadline is $d_{1}=1$, he gets the object if his virtual valuation $J_{1}\left(v_{1}\right)$ is higher than the option value of storing the object for the second period. The option value is equal to $E\left[V_{2}\left(s_{2}\right) \mid d_{1}=1, k_{2}=1\right]=E\left[\max \left\{0, J_{2}\left(v_{2}\right)\right\}\right]$. Therefore, the 
interim winning probability is given by

$$
q_{1}^{\mathrm{rlx}}\left(v_{1}, d_{1}=1\right)= \begin{cases}1, & \text { if } J_{1}\left(v_{1}\right) \geq E\left[\max \left\{0, J_{2}\left(v_{2}\right)\right\}\right], \\ 0, & \text { otherwise }\end{cases}
$$

If, on the other hand, the deadline of the first buyer is $d_{1}=2$, the object is stored and he wins in the second period if his virtual valuation is positive and greater than the virtual valuation of the second buyer. Therefore, his interim winning probability is given by

$$
q_{1}^{\mathrm{rlx}}\left(v_{1}, d_{1}=2\right)=\operatorname{Prob}\left[J_{1}\left(v_{1}\right) \geq \max \left\{0, J_{2}\left(v_{2}\right)\right\}\right] .
$$

Since we assume that the valuations of both buyers are drawn from distributions with the same support, there exists a valuation $\tilde{v}_{1}<\bar{v}$, such that $J_{1}\left(\tilde{v}_{1}\right)=E\left[\max \left\{0, J_{2}\left(v_{2}\right)\right\}\right]$. Therefore, for all valuations $v_{1}>\tilde{v}_{1}$, we have $q_{1}^{\mathrm{rlx}}\left(v_{1}, d_{1}=1\right)=1$. If $q_{1}^{\mathrm{rlx}}(v, d)$ is nondecreasing in $d$, we must therefore also have

$$
q_{1}^{\mathrm{rlx}}\left(v_{1}, d_{1}=2\right)=\operatorname{Prob}\left[J_{1}\left(v_{1}\right) \geq \max \left\{0, J_{2}\left(v_{2}\right)\right\}\right]=1,
$$

for all $v_{1}>\tilde{v}_{1}$. On the other hand, $J_{1}\left(v_{1}\right)<\bar{v}$ implies that $\operatorname{Prob}\left[J_{1}\left(v_{1}\right) \geq \max \left\{0, J_{2}\left(v_{2}\right)\right\}\right]<$ 1 , since $F_{2}$ has support $[0, \bar{v}]$. Therefore, $q^{\text {rlx }}$ cannot be non-decreasing in $d$ for all $v .^{45}$

The example demonstrates that monotonicity with respect to the deadline is unlikely to be satisfied because in period one, there is uncertainty about the valuation of the buyer who arrives in the second period. Consequently, the allocation decision in the first period is only based on the realized value of buyer 1's valuation and expectations about future virtual valuations. Therefore, $q_{1}^{\mathrm{rlx}}\left(v_{1}, d_{1}=1\right)$ takes only extreme values zero and one. In the second period, the allocation decision takes into account the realized value of $v_{2}$. Therefore, $q_{1}^{\text {rlx }}\left(v_{1}, d_{1}=2\right)$ typically takes intermediate values in $(0,1)$. Consequently, $q_{1}^{\mathrm{rlx}}\left(v_{1}, d\right)$ is increasing in $d$ for low values of $v_{1}$, and decreasing in $d$ for high values of $v$. It is easy to see that Lemma 2 generalizes the insights from this example, because the winning probability of a buyer is closely related to the distribution function of the critical virtual valuation.

\footnotetext{
$\overline{45}$ Note that this argument does not depend on the assumption that $v$ and $d$ are conditionally independent as long as the support of $F_{a}(\cdot \mid d)$ is the same for all $a$ and $d$.
} 